\title{
NONLINEAR COUNTERPROPAGATING WAVES, MULTISYMPLECTIC GEOMETRY, AND THE INSTABILITY OF STANDING WAVES*
}

\author{
THOMAS J. BRIDGES ${ }^{\dagger}$ AND FIONA E. LAINE-PEARSON ${ }^{\dagger}$
}

\begin{abstract}
Standing waves are a fundamental class of solutions of nonlinear wave equations with a spatial reflection symmetry, and they routinely arise in optical and oceanographic applications. At the linear level they are composed of two synchronized counterpropagating periodic traveling waves. At the nonlinear level, they can be defined abstractly by their symmetry properties. In this paper, general aspects of the modulational instability of standing waves are considered. This problem has difficulties that do not arise in the modulational instability of traveling waves. Here we propose a new geometric formulation for the linear stability problem, based on embedding the standing wave in a four-parameter family of nonlinear counterpropagating waves. Multisymplectic geometry is shown to encode the stability properties in an essential way. At the weakly nonlinear level we obtain the surprising result that standing waves are modulationally unstable only if the component traveling waves are modulation unstable. Systems of nonlinear wave equations will be used for illustration, but general aspects will be presented, applicable to a wide range of Hamiltonian PDEs, including water waves.
\end{abstract}

Key words. modulation instability, variational principles, periodic waves, hyperbolic PDEs, water waves

AMS subject classifications. 70H33, 70S05, 76B07

DOI. $10.1137 /$ S0036139903423753

1. Introduction. When considering spatially periodic solutions of nonlinear wave equations on the real line, there are two "canonical" classes of temporally periodic solutions: traveling waves and standing waves. Standing waves arise naturally when the system has a reflection symmetry. In this paper the linear stability problem for standing waves is considered.

To illustrate the basic issues, consider the prototype nonlinear wave equation

$$
\mathbf{u}_{t t}-\mathbf{C u}_{x x}+\nabla V(\mathbf{u})=0, \quad \mathbf{u} \in \mathbb{R}^{m}, \quad x \in \mathbb{R},
$$

where $\mathbf{C}$ is a symmetric, positive definite, $m \times m$ matrix; $V: \mathbb{R}^{m} \rightarrow \mathbb{R}$ is a given smooth function; and $\nabla$ is the standard gradient on $\mathbb{R}^{m}$. This class of wave equations appears in a wide range of applications. An example is DNA modeling [30], where a typical case would be $m=2, \mathbf{C}=\operatorname{diag}\left(1, c^{2}\right)$, and $V(\mathbf{u})=\cos \left(u_{1}+u_{2}\right)-2 \cos u_{1}-2 \cos u_{2}$.

For the system (1.1) a standing wave is a spatially periodic and temporally periodic solution which is invariant under reflection $x \mapsto-x$. (A precise definition of standing wave will be given in section 3.)

Suppose a standing wave solution of (1.1) exists and denote it by $\hat{\mathbf{u}}(x, t)$. This existence problem is itself highly nontrivial due to the potential for small divisors. (The relevance of this issue is discussed in section 7.) The linearized stability equation for $\hat{\mathbf{u}}$ is then $\mathbf{u}_{t t}-\mathbf{u}_{x x}+D^{2} V(\hat{\mathbf{u}}) \mathbf{u}=0$. A modulational instability is a solution of the type $\mathbf{u}(x, t)=\operatorname{Re}\left(\mathrm{e}^{i \alpha x} \mathbf{v}(x, t)\right)$, where $\mathbf{v}(x, t)$ is periodic in $x$ of the same period as $\hat{\mathbf{u}}(x, t)$ and $\alpha$ is real with $0<|\alpha|<<1$, and $\|\mathbf{v}\|$ is exponentially growing in

\footnotetext{
* Received by the editors February 26, 2003; accepted for publication (in revised form) February 12, 2004; published electronically September 2, 2004.

http://www.siam.org/journals/siap/64-6/42375.html

${ }^{\dagger}$ Department of Mathematics and Statistics, University of Surrey, Guildford, Surrey, GU2 7XH, UK (t.bridges@eim.surrey.ac.uk).
} 
time. Basic technical issues are associated with this instability problem, such as an appropriate function space in which to define the spectral problem, but these issues will not be considered here. There is a more fundamental issue associated with modulational instability that arises even when we suppose that the basic state $\hat{\mathbf{u}}(x, t)$ is a classical solution of (1.1) and a smooth function of the wavenumber and frequency. It is this fundamental issue, which can be attributed to the fact that standing waves are related to a pair of synchronized counterpropagating traveling waves, that we will address here.

Before considering the counterpropagation property of standing waves, it is worth recalling the analogous linear stability problem for traveling waves. Let $\mathbf{u}(x, t)=\hat{\boldsymbol{\phi}}(\theta)$ be a periodic traveling wave solution of (1.1), where $\theta=\omega t+k x+\theta_{0}$. The solution $\hat{\phi}$ is a $2 \pi$-periodic function of $\theta, \omega$ is the frequency, and $k$ is the wavenumber. The distinction between stability of traveling waves and standing waves can already be seen at small amplitude. Therefore consider two well-known methods for determining whether the traveling wave is modulationally unstable: the Whitham theory [29] and the use of modulation equations such as the nonlinear Schrödinger (NLS) equation.

According to the Whitham modulation theory, a weakly nonlinear wave of amplitude $A$, of a nonlinear wave equation that can be derived from a Lagrangian formulation, is modulationally unstable if

$$
\omega_{0}^{\prime \prime}(k) \omega_{2}(k)<0,
$$

where $\omega_{0}(k)$ is the frequency of the linearized wave and $\omega_{2}(k)$ is the weakly nonlinear correction to the frequency, that is, $\omega(k)=\omega_{0}(k)+\omega_{2}(k)|A|^{2}+\cdots[29]$.

Using formal asymptotic methods, an NLS equation can be derived for the weakly nonlinear amplitude $A(X, T)$, by letting $\hat{\phi}(\theta)=A(X, T) \mathrm{e}^{\theta}+$ c.c. $+\cdots$,

$$
\mathrm{i} A_{T}+\frac{1}{2} \omega_{0}^{\prime \prime}(k) A_{X X}=\sigma|A|^{2} A
$$

(cf. [28]; see also [16] for a rigorous justification of this approach for scalar nonlinear wave equations). The basic weakly nonlinear traveling wave is represented in this equation as a solution of the form $A(X, T)=A_{0} \mathrm{e}^{\mathrm{i} \omega T}, A_{0} \in \mathbb{C}$, and this state is linearly unstable precisely when (1.2) is satisfied.

Now, the modulational instability of traveling waves, particularly the weakly nonlinear limit, is well understood, from physical, numerical, and rigorous points of view.

The case of standing waves is more difficult. Surprisingly, there is no generalization of the Whitham theory to treat the modulational instability of standing waves. The only theory in the literature that has been proposed for the modulation instability of standing waves is the use of modulation equations (Knobloch and Pierce [18]; see also [17]).

At the linear level, standing waves reduce to a pair of synchronized counterpropagating waves. Therefore one might suspect that a pair of nonlinearly coupled NLS equations of the form

$$
\begin{aligned}
& \mathrm{i} A_{T}+\mathrm{i} c_{g} A_{X}=\frac{1}{2} \omega_{0}^{\prime \prime}(k) A_{X X}-\sigma|A|^{2} A+2 \sigma(k)|B|^{2} A, \\
& \mathrm{i} B_{T}-\mathrm{i} c_{g} B_{X}=\frac{1}{2} \omega_{0}^{\prime \prime}(k) B_{X X}-\sigma|B|^{2} B+2 \sigma(k)|A|^{2} B
\end{aligned}
$$

would be a suitable model for modulation instability of standing waves. Indeed, in equation (3.3) of Okamura [23], a coupled NLS system of this form is proposed to model the instability of standing waves. The above system was derived specifically to model standing water waves, but the argument is similar for standing waves of any 
nonlinear wave equation, although the coefficients in (1.3) would differ. A standing wave is represented in this system by a solution of the form $A=B=B_{0} \mathrm{e}^{\mathrm{i} \omega T}$.

However, Knobloch and Pierce [18] argue that this coupled set of equations is not valid, and this observation is confirmed by the rigorous analysis of Pierce and Wayne [25] and Bambusi, Carati, and Ponno [2]. They argue that the coupling term needs to be replaced by mean-field coupling terms,

$$
\begin{aligned}
\mathrm{i} A_{T}^{+} & =\frac{1}{2} \omega_{0}^{\prime \prime}(k) A_{X_{+} X_{+}}^{+}-\sigma(k)\left|A^{+}\right|^{2} A^{+}+\beta(k) \Lambda^{+}\left(A^{+}\right) A^{+}, \\
-\mathrm{i} A_{T}^{-} & =\frac{1}{2} \omega_{0}^{\prime \prime}(k) A_{X_{-} X_{-}}^{-}-\sigma(k)\left|A^{-}\right|^{2} A^{-}+\beta(k) \Lambda^{-}\left(A^{-}\right) A^{-},
\end{aligned}
$$

where $X_{ \pm}=X \mp c_{g} T$ and $\Lambda^{ \pm}\left(A^{ \pm}\right)=\frac{1}{P_{ \pm}} \int_{0}^{P_{ \pm}}\left|A^{ \pm}\right|^{2} \mathrm{~d} X_{ \pm}$.

The distinction between (1.3) and (1.4) is significant as they do not give equivalent results on modulational instability of standing waves. The rational asymptotics presented in $[18,17]$, and the rigorous theory of $[25,2]$, provide strong support for the validity of (1.4).

Modulation equations have severe limitations, however. For example, the above modulation equations are limited to weakly nonlinear standing waves. In this paper we present a new theoretical framework for studying the modulational instability of standing waves. The theory is global (i.e., not restricted to small amplitude) and is based on a new variational principle.

Restricting the new theory to small amplitude waves, it predicts the same instability as the modulation equation (1.4). Since the theory presented here is significantly different from the theory used by Okamura and Knobloch and Pierce, it provides additional support for the validity of the modulation instability predicted by (1.4). Physically, the weakly nonlinear result is quite surprising, since weakly nonlinear periodic standing waves are modulationally unstable only if the component weakly nonlinear traveling waves are unstable. However, this correspondence between the instability of traveling and standing waves will not in general carry over to finiteamplitude standing waves.

The theory here will be developed for the modulation instability of standing wave solutions of Hamiltonian PDEs. The theoretical framework has two parts: first, standing waves can be characterized by a constrained variational principle that encodes information about the linear stability problem. Second, by formulating and studying the linear stability problem directly, we show how the information from the variational principle appears explicitly in the linear stability problem. The main result is that the stability exponents for all long-wave instabilities of standing waves of any amplitude (for which they exist) are determined by the roots of a quartic polynomial whose coefficients can be determined explicitly from the existing standing wave.

The obvious variational principle for standing waves does not provide enough information about the linear stability problem. Surprisingly, we find that the natural approach is to embed the family of standing waves in a four-parameter family, initially, construct a variational principle for this larger family, and then take the limit to the original two-parameter family. The argument in favor of this approach is provided by the analogy of standing waves as synchronized counterpropagating waves: the larger parameter family provides information about how the component counterpropagating waves might break up due to instability.

Conservative PDEs can be analyzed from a Lagrangian, Hamiltonian, or multisymplectic Hamiltonian viewpoint. However, neither the Lagrangian nor the classical Hamiltonian perspective provides sufficient geometry to give abstract results - that 
is, results that rely only on the Hamiltonian structure and are independent of the particular PDE. It is the multisymplectic formulation of Hamiltonian PDEs that provides sufficient geometry for a general theory. The class of Hamiltonian PDEs that we consider in canonical form is

$$
\mathbf{M} Z_{t}+\mathbf{K} Z_{x}=\nabla S(Z), \quad Z \in \mathbb{R}^{n},
$$

where $\mathbf{M}$ and $\mathbf{K}$ are constant $n \times n$ skew-symmetric matrices and $S: \mathbb{R}^{n} \rightarrow \mathbb{R}$ is a given smooth function. An example of the multisymplectification process is given in section 2. Most Hamiltonian PDEs can be cast into this form, including water waves, and other examples can be found in $[6,7,9]$ and references therein.

Abstractly, these systems can still be characterized as Lagrangian PDEs by considering Lagrangians in the canonical form

$\mathscr{L}=\iint \mathrm{L}\left(Z, Z_{t}, Z_{x}\right) \mathrm{d} x \mathrm{~d} t \quad$ with $\quad \mathrm{L}\left(Z, Z_{t}, Z_{x}\right)=\frac{1}{2}\left\langle\mathbf{M} Z_{t}, Z\right\rangle+\frac{1}{2}\left\langle\mathbf{K} Z_{x}, Z\right\rangle-S(Z)$,

where $\langle\cdot, \cdot\rangle$ is a standard inner product on $\mathbb{R}^{n}$. This Lagrangian, however, retains all the geometry - two symplectic structures and the scalar function $S$ - of the multisymplectic formulation.

An outline of the paper is as follows. In section 2, an example is given of multisymplectification, using (1.1) as an example. In section 3 standing waves are defined and it is shown that a consequence of the definition is that the momentum is identically zero. New variational principles for standing waves and standing waves embedded in a four-parameter family of counterpropagating waves are presented in section 4 . There is an interesting connection between the geometry of $\mathbf{O}(2)$-equivariant finitedimensional Hamiltonian systems, such as the spherical pendulum, and nonlinear wave equations on the real line with periodic boundary conditions, and this connection is explored in Appendix A. The details of the stability analysis for weakly nonlinear and finite-amplitude standing waves are presented in sections 6 and 7 .

The small divisor issue that appears in the analysis of standing waves is outside the scope of this paper, but the issue is briefly discussed in section 7 . One of the main motivations for studying the modulational instability of standing waves is their importance in the water-wave problem. The theory developed here does not apply directly, but we speculate on some of the implications for water waves in section 8 .

2. Multisymplectifying systems of nonlinear wave equations. The theory for instability of standing waves will be developed for the general class of PDEs (1.5). In this section, the general class of nonlinear wave equations (1.1) will be used to illustrate the transformation to multisymplectic form. In sequence, a Lagrangian, a classical Hamiltonian, and then a multisymplectic Hamiltonian formulation of this system will be presented.

The canonical form of the Lagrangian for (1.1) is

$$
\mathscr{L}=\int_{\mathscr{V}} \mathrm{L}\left(\mathbf{u}, \mathbf{u}_{t}, \mathbf{u}_{x}\right) \mathrm{d} x \wedge \mathrm{d} t, \quad \mathrm{~L}\left(\mathbf{u}, \mathbf{u}_{t}, \mathbf{u}_{x}\right)=\frac{1}{2} \mathbf{u}_{t} \cdot \mathbf{u}_{t}-\frac{1}{2} \mathbf{u}_{x} \cdot \mathbf{C} \mathbf{u}_{x}-\mathrm{V}(\mathbf{u}),
$$

where $\mathscr{V}$ represents the volume in $(x, t)$ space, and $\cdot$ represents the standard inner product on $\mathbb{R}^{m}$.

The canonical Hamiltonian formulation for the nonlinear wave equation is obtained by taking the Legendre transform with respect to time only, $\mathbf{v}=\frac{\partial \mathrm{L}}{\partial \mathbf{u}_{t}}=\mathbf{u}_{t}$, 
and then the governing equations take the form

$$
\frac{\partial}{\partial t}\left(\begin{array}{l}
\mathbf{u} \\
\mathbf{v}
\end{array}\right)=\left[\begin{array}{rr}
\mathbf{0} & \mathbf{I} \\
-\mathbf{I} & \mathbf{0}
\end{array}\right]\left(\begin{array}{l}
\delta \mathscr{H} / \delta \mathbf{u} \\
\delta \mathscr{H} / \delta \mathbf{v}
\end{array}\right), \quad \mathscr{H}(\mathbf{u}, \mathbf{v})=\int_{\mathbb{R}}\left[\frac{1}{2} \mathbf{v} \cdot \mathbf{v}+\frac{1}{2} \mathbf{u}_{x} \cdot \mathbf{C u} x+V(\mathbf{u})\right] \mathrm{d} x .
$$

This Hamiltonian formulation of the nonlinear wave equation has been widely used in analysis (see [19] and references therein). However, a disadvantage of this formulation, when studying pattern formation, is that the Hamiltonian function and symplectic structure associated with (2.2) require specification of a space of functions over the $x$ direction a priori. In the case of modulation instabilities, the basic state is periodic in space but the perturbation class will be in general quasi-periodic. In other words, we may want to determine the spatial variation of the solution set a posteriori.

Multisymplecticity puts space and time on an equal footing. The governing equations are obtained by taking a Legendre transform with respect to all directions,

$$
\mathbf{v}=\frac{\partial \mathrm{L}}{\partial \mathbf{u}_{t}}=\mathbf{u}_{t} \quad \text { and } \quad \mathbf{w}=\frac{\partial \mathrm{L}}{\partial \mathbf{u}_{x}}=-\mathbf{C} \mathbf{u}_{x}
$$

The Legendre transform generates a new Hamiltonian functional,

$$
S(\mathbf{u}, \mathbf{v}, \mathbf{w})=\mathbf{v} \cdot \mathbf{u}_{t}+\mathbf{w} \cdot \mathbf{u}_{x}-\mathrm{L}=\frac{1}{2} \mathbf{v} \cdot \mathbf{v}-\frac{1}{2} \mathbf{w} \cdot \mathbf{C}^{-1} \mathbf{w}+V(\mathbf{u}) .
$$

This function can be thought of as generated by a total Legendre transform as above, or it can be viewed as a secondary Legendre transform: $-S$ is the Legendre transform of the Hamiltonian density $H$ in (2.2).

Now, the new Lagrangian for the system is in standard form for a generalization of Hamilton's principle,

$$
\mathscr{L}=\iint \mathrm{L}(\mathbf{u}, \mathbf{v}, \mathbf{w}) \mathrm{d} x \wedge \mathrm{d} t, \quad \mathrm{~L}(\mathbf{u}, \mathbf{v}, \mathbf{w})=\mathbf{v} \cdot \mathbf{u}_{t}+\mathbf{w} \cdot \mathbf{u}_{x}-S(\mathbf{u}, \mathbf{v}, \mathbf{w}),
$$

and the governing equations are given by

$$
\begin{aligned}
& 0=\mathrm{L}_{\mathbf{u}}=-\mathbf{v}_{t}-\mathbf{w}_{x}-S_{\mathbf{u}}=-\mathbf{v}_{t}-\mathbf{w}_{x}-\nabla V(\mathbf{u}), \\
& 0=\mathrm{L}_{\mathbf{v}}=\mathbf{u}_{t}-S_{\mathbf{v}}=\mathbf{u}_{t}-\mathbf{v}, \\
& 0=\mathrm{L}_{\mathbf{w}}=\mathbf{u}_{x}-S_{\mathbf{w}}=\mathbf{u}_{x}+\mathbf{C}^{-1} \mathbf{w},
\end{aligned}
$$

using standard fixed endpoint conditions for the variations. While the PDE is now expressed as a first-order system, it has a multisymplectic structure which is awkward for analysis. It can be written in the form $\mathbf{M} Z_{x}+\mathbf{K} Z_{x}=\nabla S(Z)$ with $Z \in \mathbb{R}^{3 m}$, but the pair of symplectic operators, $\mathbf{M}$ and $\mathbf{K}$, act on $\mathbb{R}^{3 m}$ and are always degenerate. This structure can be improved by observing that $\mathbf{v}$ and $\mathbf{w}$ satisfy the constraint $\mathbf{C}^{-1} \mathbf{w}_{t}+\mathbf{v}_{x}=0$. Therefore add this constraint to the Lagrangian with vector-valued Lagrange multiplier $\mathbf{p}$, that is,

$$
\begin{aligned}
\mathscr{L} & =\int_{\mathscr{V}} \mathrm{L}(\mathbf{u}, \mathbf{v}, \mathbf{w}, \mathbf{p}) \mathrm{d} x \wedge \mathrm{d} t, \\
\mathrm{~L}(\mathbf{u}, \mathbf{v}, \mathbf{w}, \mathbf{p}) & =\mathbf{v} \cdot \mathbf{u}_{t}+\mathbf{w} \cdot \mathbf{u}_{x}-S(\mathbf{u}, \mathbf{v}, \mathbf{w})+\mathbf{p} \cdot\left(\mathbf{C}^{-1} \mathbf{w}_{t}+\mathbf{v}_{x}\right) .
\end{aligned}
$$


The governing equations are now

$$
\begin{aligned}
& 0=\mathrm{L}_{\mathbf{u}}=-\mathbf{v}_{t}-\mathbf{w}_{x}-S_{\mathbf{u}}=-\mathbf{v}_{t}-\mathbf{w}_{x}-\nabla V(\mathbf{u}), \\
& 0=\mathrm{L}_{\mathbf{v}}=\mathbf{u}_{t}-S_{\mathbf{v}}-\mathbf{p}_{x}=\mathbf{u}_{t}-\mathbf{p}_{x}-\mathbf{v}, \\
& 0=\mathrm{L}_{\mathbf{w}}=\mathbf{u}_{x}-S_{\mathbf{w}}-\mathbf{C}^{-1} \mathbf{p}_{t}=-\mathbf{C}^{-1} \mathbf{p}_{t}+\mathbf{u}_{x}+\mathbf{C}^{-1} \mathbf{w}, \\
& 0=\mathrm{L}_{\mathbf{p}}=\mathbf{C}^{-1} \mathbf{w}_{t}+\mathbf{v}_{x}=\mathbf{C}^{-1} \mathbf{w}_{t}+\mathbf{v}_{x}
\end{aligned}
$$

or

$$
\left[\begin{array}{rrcc}
0 & -\mathbf{I} & 0 & 0 \\
\mathbf{I} & 0 & 0 & 0 \\
0 & 0 & 0 & -\mathbf{C}^{-1} \\
0 & 0 & \mathbf{C}^{-1} & 0
\end{array}\right]\left(\begin{array}{c}
\mathbf{u} \\
\mathbf{v} \\
\mathbf{w} \\
\mathbf{p}
\end{array}\right)_{t}+\left[\begin{array}{cccc}
0 & 0 & -\mathbf{I} & 0 \\
0 & 0 & 0 & -\mathbf{I} \\
\mathbf{I} & 0 & 0 & 0 \\
0 & \mathbf{I} & 0 & 0
\end{array}\right]\left(\begin{array}{c}
\mathbf{u} \\
\mathbf{v} \\
\mathbf{w} \\
\mathbf{p}
\end{array}\right)_{x}=\left(\begin{array}{c}
\nabla \mathbf{V}(\mathbf{u}) \\
\mathbf{v} \\
-\mathbf{C}^{-1} \mathbf{w} \\
0
\end{array}\right)
$$

This system can be expressed in canonical multisymplectic form (1.5) with $n=4 m$, and indeed, in this case, $\mathbf{M}$ and $\mathbf{K}$ define symplectic structures on $\mathbb{R}^{4 m}$. The two symplectic structures do not commute in general, unless $\mathbf{C}=\mathbf{I}$, since

$$
[\mathbf{M}, \mathbf{K}]=\mathbf{M K}-\mathbf{K M}=\left[\begin{array}{cccc}
0 & 0 & 0 & 1 \\
0 & 0 & -1 & 0 \\
0 & -1 & 0 & 0 \\
1 & 0 & 0 & 0
\end{array}\right] \otimes\left(\mathbf{I}-\mathbf{C}^{-1}\right)
$$

In the scalar case $m=1$, scaling can be introduced so that $\mathbf{M}$ and $\mathbf{K}$ always commute.

In summary, the main point of this section is that the system of nonlinear wave equations (1.1) can be characterized in terms of geometric properties: two symplectic structures, and a scalar-valued function $S(Z)$, on a finite-dimensional phase space: $Z \in \mathbb{R}^{n}$.

A property of the nonlinear wave equation (1.1) that is important for the existence of standing waves is reversibility in $x$. If $\mathbf{u}(x, t)$ is a solution of $(1.1)$, then $\mathbf{u}(-x, t)$ is also a solution. In the multisymplectification of (1.1), this reversibility is represented by the action

$$
\mathbf{r} \cdot Z(x, t)=\mathbf{R} Z(-x, t) \quad \text { with } \quad \mathbf{R}=\operatorname{diag}(\mathbf{I}, \mathbf{I},-\mathbf{I},-\mathbf{I}) \in \mathbb{R}^{4 m \times 4 m}
$$

The involution $\mathbf{R}$ and its associated action satisfy

$$
\mathbf{R M}=\mathbf{M R}, \quad \mathbf{R K}=-\mathbf{K R}, \quad \text { and } \quad S(\mathbf{r} \cdot Z)=S(Z) .
$$

In turn, the properties (2.7) imply that $\mathbf{r} \cdot Z$ is a solution of the wave equation in the form (1.5) whenever $Z$ is.

The system of nonlinear wave equations (1.1) is reversible in $t$ as well, and a multisymplectic $t$-reversor can also be defined, but $t$-reversibility will not be needed in the general theory for standing waves. 
3. Standing wave solutions of Hamiltonian PDEs. The theory for standing waves can be developed based only on the geometric properties of the multisymplectic formulation. Therefore, as in the previous section, we will assume that the PDE has been transformed to a multisymplectic Hamiltonian PDE, and we take the following general class of PDEs as the starting point for the analysis:

$$
\mathbf{M} Z_{t}+\mathbf{K} Z_{x}=\nabla S(Z), \quad Z \in \mathbb{R}^{n} .
$$

The only hypotheses on (3.1) are that $\mathbf{M}$ and $\mathbf{K}$ are constant $n \times n$ skew-symmetric matrices and $S: \mathbb{R}^{n} \rightarrow \mathbb{R}$ is a given smooth function (at least twice continuously differentiable), which does not depend explicitly on $x$ or $t$. On $\mathbb{R}^{n}$, the standard inner product will be denoted by $\langle\cdot, \cdot\rangle$.

For the existence of standing waves, we will require that the system (3.1) is $x$ reversible with a multisymplectic action of the reversor,

$$
\mathbf{r} \cdot Z(x, t)=\mathbf{R} Z(-x, t)
$$

for some isometric involution $\mathbf{R}: \mathbb{R}^{n} \rightarrow \mathbb{R}^{n}$ satisfying the identities (2.7) (with $\mathbf{M}$, K and $S$ associated with (3.1)). In this setting, an abstract definition of a standing wave can be given.

Definition. A solution $\widehat{Z}(x, t)$ of (3.1) is called a standing wave if it is periodic in both $x$ and $t$ and satisfies $\mathbf{r} \cdot \widehat{Z}(x, t)=\widehat{Z}(x, t)$.

Curiously, we cannot find anywhere in the literature where a general definition of standing waves for nonlinear PDEs has heretofore been given.

It is sometimes remarked that standing waves are spatially periodic waves with zero momentum. However, we can show that zero momentum is a consequence of the above definition.

What is momentum? The momentum here is defined to be the conserved quantity given by Noether's theorem associated with the translation invariance in $x$ of the PDE. If (3.1) represents a physical system, this conserved quantity may indeed be the physical momentum. An application of Noether's theorem to the Lagrangian (1.6) (see Appendix B for this argument) shows that the appropriate form for the momentum on a space of functions that are $2 \pi$ periodic in $x$ is

$$
\mathscr{I}(Z)=\oint \frac{1}{2}\left\langle\mathbf{M} Z_{x}(x, t), Z(x, t)\right\rangle \mathrm{d} x \quad \text { where } \quad \oint() \mathrm{d} x:=\frac{1}{2 \pi} \int_{0}^{2 \pi}(\mathrm{)} \mathrm{d} x .
$$

Given this expression for momentum, we can show that $\mathscr{I}(\widehat{Z})=0$ if $\widehat{Z}(x, t)$ is a standing wave solution of (3.1):

$$
\begin{aligned}
\mathscr{I}(\mathbf{r} \cdot Z)= & \oint \frac{1}{2}\left\langle\mathbf{M R}(Z(-x, t))_{x}, \mathbf{R} Z(-x, t)\right\rangle \mathrm{d} x \quad \text { (by definition) } \\
= & -\oint \frac{1}{2}\left\langle\mathbf{M R} Z_{x}(-x, t), \mathbf{R} Z(-x, t)\right\rangle \mathrm{d} x \\
= & \left.-\oint \frac{1}{2}\left\langle\mathbf{R} \mathbf{M} Z_{x}(-x, t), \mathbf{R} Z(-x, t)\right\rangle \mathrm{d} x \quad \text { (using } \mathbf{R M}=\mathbf{M R}\right) \\
= & -\oint \frac{1}{2}\left\langle\mathbf{M} Z_{x}(-x, t), Z(-x, t)\right\rangle \mathrm{d} x \quad \text { (since } \mathbf{R} \text { is an isometry) } \\
= & -\oint \frac{1}{2}\left\langle\mathbf{M} Z_{x}(x, t), Z(x, t)\right\rangle \mathrm{d} x \\
& (\text { using the change of variable } x \mapsto-x \text { and periodicity) } \\
= & -\mathscr{I}(Z) .
\end{aligned}
$$


Therefore, if $\widehat{Z}(x, t)$ is a standing wave and so $\mathbf{r} \cdot \widehat{Z}=\widehat{Z}$, it is immediate that $\mathscr{I}(\widehat{Z})=0$.

4. Variational principles for standing waves and counterpropagating waves. At the linear level, a standing wave consists of a pair of synchronized counterpropagating waves,

$$
Z(x, t)=A \xi \mathrm{e}^{\mathrm{i}(\omega t+k x)}+A \xi \mathrm{e}^{\mathrm{i}(\omega t-k x)}+\text { c.c. },
$$

where $A \in \mathbb{C}$ is a complex amplitude and $\xi \in \mathbb{C}^{n}$ is an eigenvector associated with the linearization of (3.1). A natural generalization of this form to finite amplitude is to look for nonlinear solutions of the form

$$
Z(x, t)=\widehat{Z}\left(\theta_{1}, \theta_{2}\right), \quad \theta_{1}=\omega t+k x+\theta_{1}^{o}, \quad \theta_{2}=\omega t-k x+\theta_{2}^{o},
$$

where $\theta_{j}^{o}$ are arbitrary constant phases and $\widehat{Z}$ is a $2 \pi$-periodic function of $\theta_{1}$ and $\theta_{2}$.

Substituting the form (4.1) into (3.1) results in

$$
\omega \mathbf{M}\left(\frac{\partial \widehat{Z}}{\partial \theta_{1}}+\frac{\partial \widehat{Z}}{\partial \theta_{2}}\right)+k \mathbf{K}\left(\frac{\partial \widehat{Z}}{\partial \theta_{1}}-\frac{\partial \widehat{Z}}{\partial \theta_{2}}\right)=\nabla S(\widehat{Z}) .
$$

The operators $\mathbf{M} \partial_{\theta_{j}}$ and $\mathbf{K} \partial_{\theta_{j}}$ are formally self-adjoint operators on a space of doubly periodic functions. Hence, treating $\omega$ and $k$ as Lagrange multipliers, (4.2) can be interpreted as the necessary condition for a constrained variational principle. Let

$$
\mathscr{A}(Z)=\int_{\mathbb{T}^{2}} \frac{1}{2}\left\langle\mathbf{M}\left(\partial_{\theta_{1}}+\partial_{\theta_{2}}\right) Z, Z\right\rangle \mathrm{d} \theta \quad \text { and } \quad \mathscr{B}(Z)=\int_{\mathbb{T}^{2}} \frac{1}{2}\left\langle\mathbf{K}\left(\partial_{\theta_{1}}-\partial_{\theta_{2}}\right) Z, Z\right\rangle \mathrm{d} \theta,
$$

where $\int_{\mathbb{T}^{2}}() \mathrm{d} \theta:=(2 \pi)^{-2} \int_{0}^{2 \pi} \int_{0}^{2 \pi}() \mathrm{d} \theta_{1} \mathrm{~d} \theta_{2}$. The constrained variational principle is then to find critical points of $\mathscr{S}, S(Z)$ averaged over $\mathbb{T}^{2}$, subject to fixed values of the constraints $\mathscr{A}$ and $\mathscr{B}$. It follows from standard Lagrange multiplier theory that this constrained variational principle is nondegenerate when

$$
\operatorname{det}\left[\begin{array}{cc}
\mathscr{A}_{\omega} & \mathscr{A}_{k} \\
\mathscr{B}_{\omega} & \mathscr{B}_{k}
\end{array}\right] \neq 0
$$

This variational principle gives a global characterization of any state of (3.1) which is periodic in both $x$ and $t$. It includes a characterization of standing waves and traveling waves. The special case of strictly traveling waves was considered in [7], and it is shown there that the sign of the determinant (4.3) carries information about linear stability.

Another way to view this variational principle is as a generalization to the spatiotemporal setting of the classical variational principle for periodic solutions of finitedimensional Hamiltonian systems: find critical points of the energy (Hamiltonian) on level sets of the action on a space of periodic functions, with the frequency $\omega$ as a Lagrange multiplier. For finite-dimensional Hamiltonian systems this variational principle has been widely used to prove the existence of periodic solutions (cf. [20] and references therein). However, the variational principle associated with (4.1) is more difficult to work with for the case of standing waves. Although standing waves are periodic solutions, the fact that there is an infinite number of modes can cause problems with small divisors (see section 7 ). 
The form of the solution (4.1) is not the most general form for a pair of counterpropagating waves. When considering the linear stability problem for standing waves, it will turn out that a somewhat more general variational principle will be crucial for getting a geometric characterization of linear instability of standing waves. The idea is to embed the family of standing waves in a four-parameter family of counterpropagating waves, with the standing wave obtained as a limiting two-parameter case.

Consider the more general class of solutions of (3.1); let

$$
Z(x, t)=\widehat{Z}\left(\theta_{1}, \theta_{2}\right) \quad \text { with } \quad \theta_{j}=\omega_{j} t+k_{j} x+\theta_{j}^{o}, \quad j=1,2,
$$

where $\widehat{Z}$ is again a $2 \pi$-periodic function of both $\theta_{1}$ and $\theta_{2}$. The significant difference here is that the state $\widehat{Z}$ now depends on four parameters, and the interpretation as two counterpropagating waves that are not necessarily synchronized is now evident. Indeed, in general, they may even be propagating in the same direction. However, it is the case of counterpropagating waves, near synchronized standing waves, that is of greatest interest here, that is, $k_{1}+k_{2} \approx 0$ and $\omega_{1}-\omega_{2} \approx 0$.

The function $\widehat{Z}$ now satisfies

$$
\omega_{1} \mathbf{M} \frac{\partial \widehat{Z}}{\partial \theta_{1}}+\omega_{2} \mathbf{M} \frac{\partial \widehat{Z}}{\partial \theta_{2}}+k_{1} \mathbf{K} \frac{\partial \widehat{Z}}{\partial \theta_{1}}+k_{2} \mathbf{K} \frac{\partial \widehat{Z}}{\partial \theta_{2}}=\nabla \mathscr{S}(\widehat{Z}),
$$

where $\mathscr{S}$ is $S$ averaged over $\theta_{1}$ and $\theta_{2}$. Equation (4.5) can be interpreted as the Lagrange necessary condition for the constrained variational principle: find critical points of $S$ averaged over $\mathbb{T}^{2}$ restricted to level sets of the four functionals

$$
\mathscr{A}_{j}(Z)=\int_{\mathbb{T}^{2}} \frac{1}{2}\left\langle\mathbf{M} \partial_{\theta_{j}} Z, Z\right\rangle \mathrm{d} \theta \quad \text { and } \quad \mathscr{B}_{j}(Z)=\int_{\mathbb{T}^{2}} \frac{1}{2}\left\langle\mathbf{K} \partial_{\theta_{j}} Z, Z\right\rangle \mathrm{d} \theta, \quad j=1,2
$$

The Lagrange necessary condition can be written

$$
\nabla \mathscr{S}(\widehat{Z})=\omega_{1} \nabla \mathscr{A}_{1}(\widehat{Z})+\omega_{2} \nabla \mathscr{A}_{2}(\widehat{Z})+k_{1} \nabla \mathscr{B}_{1}(\widehat{Z})+k_{2} \nabla \mathscr{B}_{2}(\widehat{Z}) .
$$

The frequencies $\omega_{1}, \omega_{2}$ and the wavenumbers $k_{1}, k_{2}$ appear as Lagrange multipliers. Using standard Lagrange multiplier theory, this constrained variational principle is nondegenerate if

$$
\operatorname{det}\left[\begin{array}{cc}
\frac{\delta \mathscr{A}}{\delta \omega} & \frac{\delta \mathscr{A}}{\delta k} \\
\frac{\delta \mathscr{B}}{\delta \omega} & \frac{\delta \mathscr{B}}{\delta k}
\end{array}\right] \neq 0, \quad \text { where } \quad \frac{\delta \mathscr{A}}{\delta \omega}=\left(\begin{array}{ll}
\frac{\partial \mathscr{A}_{1}}{\partial \omega_{1}} & \frac{\partial \mathscr{A}_{1}}{\partial \omega_{2}} \\
\frac{\partial \mathscr{A}_{2}}{\partial \omega_{1}} & \frac{\partial \mathscr{A}_{2}}{\partial \omega_{2}}
\end{array}\right)
$$

with similar expressions for the $2 \times 2$ matrices $\frac{\delta \mathscr{A}}{\delta k}, \frac{\delta \mathscr{B}}{\delta \omega}, \frac{\delta \mathscr{B}}{\delta k}$. It is the two-parameter subfamily of two-wave interactions that correspond to standing waves that is of interest. Given a function $\widehat{Z}\left(\theta_{1}, \theta_{2}\right)$ satisfying this variational principle, a standing wave is recovered formally by taking the limit to synchronized counterpropagating waves

$$
\omega_{1} \rightarrow \omega, \quad k_{1} \rightarrow k, \quad \omega_{2} \rightarrow \omega, \quad \text { and } \quad k_{2} \rightarrow-k
$$

if the limits exist. This limit is taken after the Jacobian matrices in (4.8) are computed.

At first sight, this limit might seem a bit questionable: taking the limit on a torus from irrational values to a resonance? However, there is additional structure 
here. The translation invariance in $x$ restricted to periodic functions along with the $x$-reversibility generates the group $\mathbf{O}(2)$. Translation invariance in time restricted to periodic functions generates an action of $\mathbb{S}^{1}$. Combining these groups gives $\mathbf{O}(2) \times \mathbb{S}^{1}$ : a toral symmetry. The toral symmetry is almost enough structure to allow for smooth variation of parameters on the torus. Indeed, if the system was finite-dimensional, this would be true, and this case is discussed briefly in Appendix A. The obstacle to smoothness for the above limit leading to standing waves is again the potential for small divisors due to a countable number of purely imaginary eigenvalues (see section 7).

5. Stability analysis of nonlinear standing waves. It is in the study of the stability of standing waves that the importance of the embedding of standing waves in the four-parameter family becomes apparent. In this section the linear stability problem for standing waves is formulated and it is shown that the entries in the determinant (4.8) appear in the linear stability analysis in a central way. The strategy is to linearize (3.1) about the full four-parameter two-wave interaction. Then, after the stability condition is deduced, the limit to standing waves is taken.

Substitute $Z(x, t)=\widehat{Z}\left(\theta_{1}, \theta_{2}\right)+\widehat{U}\left(\theta_{1}, \theta_{2}, x, t\right)$, where $\widehat{Z}$ is the wave (4.4) and $\widehat{U}$ is a perturbation, into (3.1) and linearize about $\widehat{Z}$,

$$
\mathbf{M} \widehat{U}_{t}+\mathbf{K} \widehat{U}_{x}=\mathbf{L}\left(\theta_{1}, \theta_{2}\right) \widehat{U},
$$

where

$$
\begin{aligned}
\mathbf{L}\left(\theta_{1}, \theta_{2}\right) & =D^{2} \mathscr{S}(\widehat{Z})-\mathbf{M}\left[\omega_{1} \frac{\partial}{\partial \theta_{1}}+\omega_{2} \frac{\partial}{\partial \theta_{2}}\right]-\mathbf{K}\left[k_{1} \frac{\partial}{\partial \theta_{1}}+k_{2} \frac{\partial}{\partial \theta_{2}}\right] \\
& =D^{2} \mathscr{S}(\widehat{Z})-\omega_{1} D^{2} \mathscr{A}_{1}(\widehat{Z})-\omega_{2} D^{2} \mathscr{A}_{2}(\widehat{Z})-k_{1} D^{2} \mathscr{B}_{1}(\widehat{Z})-k_{2} D^{2} \mathscr{B}_{2}(\widehat{Z}) .
\end{aligned}
$$

The operator $\mathbf{L}$ is a linear partial differential operator with nonconstant (periodic) coefficients depending on $\theta_{1}$ and $\theta_{2}$. Introduce a class of perturbations of modulation type

$$
\widehat{U}\left(\theta_{1}, \theta_{2}, x, t\right)=\operatorname{Re}\left(U\left(\theta_{1}, \theta_{2}\right) \mathrm{e}^{\lambda t+\mathrm{i} \alpha x}\right),
$$

where $\lambda \in \mathbb{C}$ is the stability exponent and $\alpha \in \mathbb{R}$ is the modulation parameter associated with the $x$-direction. The eigenvalue problem for $\left(\lambda, U\left(\theta_{1}, \theta_{2}\right)\right)$ is then

$$
\mathbf{L}\left(\theta_{1}, \theta_{2}\right) U=\lambda \mathbf{M} U+\mathrm{i} \alpha \mathbf{K} U .
$$

DEFINITION. If there exists a solution $U\left(\theta_{1}, \theta_{2}\right)$ of (5.3) which is $2 \pi$-periodic in $\theta_{1}$ and $\theta_{2}$, for some $\lambda \in \mathbb{C}$ and $\alpha \in \mathbb{R}$, with $\operatorname{Re}(\lambda)>0$, then we say that the basic state $\widehat{Z}\left(\theta_{1}, \theta_{2}\right)$ is linearly unstable or spectrally unstable.

The application of this definition and the development of the geometric stability condition are not rigorous. For example, identification of the precise space of functions in which $U\left(\theta_{1}, \theta_{2}\right)$ might exist is beyond the scope of this paper. The obstacle to rigor is the potential small divisor problem, which would result in the range of the operator $\mathbf{L}$ not being closed. In some special cases, for example, if there is enough symmetry [9], the theory can be made rigorous using a Lyapunov-Schmidt reduction.

The eigenvalue problem (5.3) is still a PDE in $\theta_{1}$ and $\theta_{2}$. It has considerable structure (combination of symmetric and antisymmetric operators), but we do not 
expect to be able to analyze this spectral problem completely. However, we can get complete results on long-wave instability, that is, when $|\alpha| \ll 1$. In this case, the geometry of (4.4) can be used to give a geometric characterization of the spectrum for $\alpha$ small. Eigenvalue problems of this type have been studied geometrically before in a different but related context [9, section 5], and therefore we can appeal to those results.

The kernel of $\mathbf{L}\left(\theta_{1}, \theta_{2}\right)$ has (at least) two elements,

$$
\operatorname{Ker}(\mathbf{L}) \supseteq \operatorname{span}\left\{\frac{\partial \widehat{Z}}{\partial \theta_{1}}, \frac{\partial \widehat{Z}}{\partial \theta_{2}}\right\},
$$

and this can be verified by differentiating (4.7) with respect to $\theta_{1}$ and $\theta_{2}$. Assume that these two functions are the only elements in the kernel, a property that is generically satisfied. (For certain parameter values, the kernel might be larger.) Then look for long-wave instabilities $\alpha \ll 1$ by expanding $U$ in a Taylor series. Consider the ansatz

$$
U=c_{1}\left(\widehat{Z}_{\theta_{1}}+\lambda \widehat{Z}_{\omega_{1}}+\mathrm{i} \alpha \widehat{Z}_{k_{1}}\right)+c_{2}\left(\widehat{Z}_{\theta_{2}}+\lambda \widehat{Z}_{\omega_{1}}+\mathrm{i} \alpha \widehat{Z}_{k_{2}}\right)+\mathcal{O}\left(|\lambda|^{2}+|\alpha|^{2}\right),
$$

where $\mathbf{c}=\left(c_{1}, c_{2}\right)$ are arbitrary complex constants. By differentiating (4.7) with respect to $\omega_{1}, \omega_{2}, k_{1}$, and $k_{2}$ it can be verified that this expression is indeed the solution to (5.3) to leading order.

It is worth remarking here that it is precisely in the leading-order expression for $U$ that the deformation from standing waves to the general two-wave interaction is necessary. Four derivatives of $\widehat{Z}$ with respect to parameters are needed in (5.5), whereas if there was just one frequency and one wavenumber, only $\widehat{Z}_{\omega}$ and $\widehat{Z}_{k}$ would be available for (5.5).

Since $\mathbf{L}$ is formally self-adjoint, the solvability condition for (5.3) is

$$
\begin{aligned}
& \llbracket \widehat{Z}_{\theta_{1}}, \lambda \mathbf{M} U+\mathrm{i} \alpha \mathbf{K} U \rrbracket=0, \\
& \llbracket \widehat{Z}_{\theta_{2}}, \lambda \mathbf{M} U+\mathrm{i} \alpha \mathbf{K} U \rrbracket=0,
\end{aligned}
$$

where

$$
\llbracket f, g \rrbracket=\int_{\mathbb{T}^{2}}\left\langle f\left(\theta_{1}, \theta_{2}\right), g\left(\theta_{1}, \theta_{2}\right)\right\rangle \mathrm{d} \theta=\frac{1}{(2 \pi)^{2}} \int_{0}^{2 \pi} \int_{0}^{2 \pi}\left\langle f\left(\theta_{1}, \theta_{2}\right), g\left(\theta_{1}, \theta_{2}\right)\right\rangle \mathrm{d} \theta_{1} \mathrm{~d} \theta_{2} .
$$

This solvability condition still contains the unknown function $U$, but we have a leading-order expression for $U$. Substituting the leading-order expression for $U$ into the solvability condition leads to the pair of algebraic equations

$$
\left[\mathbf{N}_{0} \lambda^{2}+\mathrm{i} \alpha \lambda \mathbf{N}_{1}+(\mathrm{i} \alpha)^{2} \mathbf{N}_{2}+\cdots\right]\left(\begin{array}{l}
c_{1} \\
c_{2}
\end{array}\right)=\left(\begin{array}{l}
0 \\
0
\end{array}\right),
$$

where $\mathbf{N}_{j}, j=0,1,2$, are $2 \times 2$ matrices depending only on the properties of the basic wave $\widehat{Z}$. The derivation of the expression for $\mathbf{N}_{0}$ will be given, and then the result for the other two will be stated. From the solvability condition we have that

$$
\mathbf{N}_{0}=\left[\begin{array}{ll}
\int_{\mathbb{T}^{2}}\left\langle\widehat{Z}_{\theta_{1}}, \mathbf{M} \widehat{Z}_{\omega_{1}}\right\rangle \mathrm{d} \theta & \int_{\mathbb{T}^{2}}\left\langle\widehat{Z}_{\theta_{1}}, \mathbf{M} \widehat{Z}_{\omega_{2}}\right\rangle \mathrm{d} \theta \\
\int_{\mathbb{T}^{2}}\left\langle\widehat{Z}_{\theta_{2}}, \mathbf{M} \widehat{Z}_{\omega_{1}}\right\rangle \mathrm{d} \theta & \int_{\mathbb{T}^{2}}\left\langle\widehat{Z}_{\theta_{2}}, \mathbf{M} \widehat{Z}_{\omega_{2}}\right\rangle \mathrm{d} \theta
\end{array}\right] .
$$


However, by differentiating the functionals (4.6) with respect to $\omega_{1}$ and $\omega_{2}$ we find that the matrix simplifies to

$$
\mathbf{N}_{0}=-\left[\begin{array}{ll}
\frac{\partial \mathscr{A}_{1}}{\partial \omega_{1}} & \frac{\partial \mathscr{A}_{1}}{\partial \omega_{2}} \\
\frac{\partial \mathscr{A}_{2}}{\partial \omega_{1}} & \frac{\partial \mathscr{A}_{2}}{\partial \omega_{2}}
\end{array}\right]=-\frac{\partial \mathscr{A}}{\partial \omega} .
$$

Similarly, $\mathbf{N}_{1}=-\frac{\partial \mathscr{A}}{\partial k}-\frac{\partial \mathscr{B}}{\partial \omega}$ and $\mathbf{N}_{2}=-\frac{\partial \mathscr{B}}{\partial k}$, and so (5.6) reduces to

$$
\left(\lambda^{2} \frac{\delta \mathscr{A}}{\delta \omega}+\mathrm{i} \alpha \lambda\left(\frac{\delta \mathscr{A}}{\delta k}+\frac{\delta \mathscr{B}}{\delta \omega}\right)+(\mathrm{i} \alpha)^{2} \frac{\delta \mathscr{B}}{\delta k}+\cdots\right)\left(\begin{array}{l}
c_{1} \\
c_{2}
\end{array}\right)=\left(\begin{array}{l}
0 \\
0
\end{array}\right) .
$$

Therefore, if $|\lambda|+|\alpha|$ is sufficiently small and the matrix (4.8) is nondegenerate, the long-wave stability of the basic two-wave interaction is determined by the quartic

$$
\begin{aligned}
\Delta(\lambda, \alpha) & =\operatorname{det}\left[\lambda^{2} \frac{\delta \mathscr{A}}{\delta \omega}+\mathrm{i} \alpha \lambda\left(\frac{\delta \mathscr{A}}{\delta k}+\frac{\delta \mathscr{B}}{\delta \omega}\right)+(\mathrm{i} \alpha)^{2} \frac{\delta \mathscr{B}}{\delta k}\right] \\
& =\operatorname{det}\left[\sigma^{\mathrm{T}} \otimes \mathbf{I}_{2}\left(\begin{array}{ll}
\delta \mathscr{A} / \delta \omega & \delta \mathscr{A} / \delta k \\
\delta \mathscr{B} / \delta \omega & \delta \mathscr{B} / \delta k
\end{array}\right) \sigma \otimes \mathbf{I}_{2}\right], \quad \sigma=\left(\begin{array}{c}
\lambda \\
\mathrm{i} \alpha
\end{array}\right) .
\end{aligned}
$$

The second form shows that central role played by the nondegeneracy condition from the constrained variational principle of section 4 .

Expanding out the determinant in (5.7) leads to a quartic polynomial for $\lambda$,

$$
\Delta(\lambda, \alpha)=a_{4} \lambda^{4}+a_{3} \lambda^{3}+a_{2} \lambda^{2}+a_{1} \lambda+a_{0}
$$

with

$$
\begin{aligned}
& a_{4}=\operatorname{det}\left(\frac{\delta \mathscr{A}}{\delta \omega}\right), \\
& a_{3}=\mathrm{i} \alpha \operatorname{Tr}\left(\frac{\delta \mathscr{A}}{\delta \omega}^{\#}\left(\frac{\delta \mathscr{A}}{\delta k}+\frac{\delta \mathscr{B}}{\delta \omega}\right)\right), \\
& a_{2}=-\alpha^{2} \operatorname{det}\left(\frac{\delta \mathscr{A}}{\delta k}+\frac{\delta \mathscr{B}}{\delta \omega}\right)-\alpha^{2} \operatorname{Tr}\left(\frac{\delta \mathscr{A}^{\#}}{\delta \omega} \frac{\delta \mathscr{B}}{\delta k}\right), \\
& a_{1}=-\mathrm{i} \alpha^{3} \operatorname{Tr}\left(\frac{\delta \mathscr{B}}{\delta k} \frac{\delta \mathscr{A}}{\delta k}+\frac{\delta \mathscr{B}}{\delta \omega}\right), \\
& a_{0}=\alpha^{4} \operatorname{det}\left(\frac{\delta \mathscr{B}}{\delta k}\right),
\end{aligned}
$$

where the superscript \# indicates adjugate, i.e.,

$$
\mathbf{C}^{\#}=\left(\begin{array}{ll}
c_{1} & c_{2} \\
c_{2} & c_{3}
\end{array}\right)^{\#}=\mathbf{J}^{-1} \mathbf{C J}=\left(\begin{array}{rr}
c_{3} & -c_{2} \\
-c_{2} & c_{1}
\end{array}\right), \quad \text { where } \mathbf{J}=\left(\begin{array}{rr}
0 & -1 \\
1 & 0
\end{array}\right) .
$$

This stability quartic applies to both standing waves and the deformed two-wave interaction, which may have independent interest. Given a basic four-parameter wave, $\left(\widehat{Z} ; \omega_{1}, \omega_{2}, k_{1}, k_{2}\right)$, the coefficients of the quartic can in principle be computed, and then the quartic solved for the four roots, thereby determining whether there is a long-wave instability. 


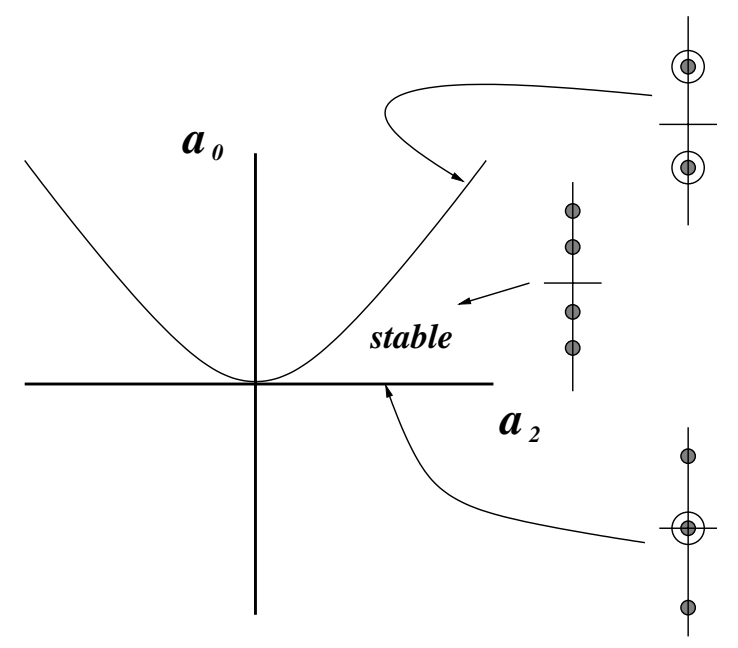

FIG. 1. Position of the roots of the quartic $\Delta(\lambda, \alpha)=0$ when $a_{1}=a_{3}=0$ and $a_{4}=1$, showing the stable region and its boundary in the $\left(a_{2}, a_{0}\right)$ plane. In all other regions there is at least one unstable root.

5.1. The stability quartic when $a_{1}=a_{3}=0$. A special case of the quartic that can be analyzed in detail is when $a_{1}=a_{3}=0$. This case will not arise in general for standing waves at finite amplitude, but it does arise in the limit as the amplitude of the wave tends to zero - the weakly nonlinear limit.

In the analysis of the stability quartic $\Delta(\lambda, \alpha)=0$, the term "instability" will mean that there is at least one root of $\Delta(\lambda, \alpha)=0$ with positive real part, and "stability" will mean that all four roots are purely imaginary and simple (spectral stability). We have the following complete classification of the roots of (5.8) when $a_{1}=a_{3}=0$ :

$$
\begin{aligned}
a_{4} a_{0}<0 & \Rightarrow \text { instability, } \\
a_{4} a_{0} \geq 0 \text { but } a_{4} a_{2}<0 & \Rightarrow \text { instability, } \\
a_{4} a_{0}>0 \text { but } a_{4} a_{2}=0 & \Rightarrow \text { instability, } \\
a_{4} a_{0}>0 \text { and } a_{4} a_{2}>0 \text { but } a_{2}^{2}-4 a_{4} a_{0}<0 & \Rightarrow \text { instability, } \\
a_{4} a_{0}>0, a_{4} a_{2}>0 \text { and } a_{2}^{2}-4 a_{4} a_{0}>0 & \Rightarrow \text { stability. }
\end{aligned}
$$

There are also two special cases where the spectrum is purely imaginary but there are multiple eigenvalues. When $a_{0} a_{4}=0, a_{2} a_{4}>0$, and $a_{2}^{2}-4 a_{4} a_{0}>0$, there is a pair of distinct purely imaginary eigenvalues and a double zero eigenvalue. When $a_{4} a_{0}>0$, $a_{4} a_{2}>0$ but $a_{2}^{2}-4 a_{4} a_{0}=0$ there is a pair of purely imaginary eigenvalues each of multiplicity two. These special cases lie on the boundary of the region of stability, as illustrated in Figure 1.

6. Instability of weakly nonlinear standing waves. The purpose of this section is threefold. It illustrates in the simplest possible setting how the variational principle and stability theory accumulate information on the spectral problem. It shows explicitly the importance of the limit from the four-parameter two-wave interaction to the two-parameter standing wave. Third, it shows that the theory of this paper recovers the modulation instability predicted by coupled NLS equations with mean-field coupling. 
To construct weakly nonlinear counterpropagating waves, take a Fourier ansatz,

$$
\begin{aligned}
\widehat{Z}\left(\theta_{1}, \theta_{2}\right)= & A_{1} \boldsymbol{\xi}_{1} \mathrm{e}^{\mathrm{i} \theta_{1}}+\overline{A_{1} \boldsymbol{\xi}_{1}} \mathrm{e}^{-\mathrm{i} \theta_{1}}+A_{2} \boldsymbol{\xi}_{2} \mathrm{e}^{\mathrm{i} \theta_{2}}+\overline{A_{2} \boldsymbol{\xi}_{2}} \mathrm{e}^{-\mathrm{i} \theta_{2}} \\
& +\Upsilon_{20}+\Upsilon_{21} \mathrm{e}^{2 \mathrm{i} \theta_{1}}+\overline{\Upsilon_{21}} \mathrm{e}^{-2 \mathrm{i} \theta_{1}}+\Upsilon_{22} \mathrm{e}^{2 \mathrm{i} \theta_{2}}+\overline{\Upsilon_{22}} \mathrm{e}^{-2 \mathrm{i} \theta_{2}} \\
& +\Upsilon_{23} \mathrm{e}^{\mathrm{i}\left(\theta_{1}+\theta_{2}\right)}+\overline{\Upsilon_{23}} \mathrm{e}^{-\mathrm{i}\left(\theta_{1}+\theta_{2}\right)}+\Upsilon_{24} \mathrm{e}^{\mathrm{i}\left(\theta_{1}-\theta_{2}\right)}+\overline{\Upsilon_{24}} \mathrm{e}^{-\mathrm{i}\left(\theta_{1}-\theta_{2}\right)}+\cdots,
\end{aligned}
$$

where $\theta_{j}=k_{j} x+\omega_{j} t(j=1,2), A_{1}$ and $A_{2}$ are complex amplitudes, and $\boldsymbol{\xi}_{1}$ and $\boldsymbol{\xi}_{2}$ have unit length. This ansatz is substituted into the Lagrangian (1.6),

$$
\begin{aligned}
\bar{L}\left(A_{1}, A_{2}, \boldsymbol{\xi}_{1}, \boldsymbol{\xi}_{2}, \mu_{1}, \mu_{2}, \Upsilon, \ldots\right)= & \mathscr{S}(\widehat{Z})-\omega_{1} \mathscr{A}_{1}-\omega_{2} \mathscr{A}_{2}-k_{1} \mathscr{B}_{1}-k_{2} \mathscr{B}_{2} \\
& -\mu_{1}\left(\left\|\boldsymbol{\xi}_{1}\right\|^{2}-1\right)-\mu_{2}\left(\left\|\boldsymbol{\xi}_{2}\right\|^{2}-1\right) .
\end{aligned}
$$

Here, $\mu_{1}$ and $\mu_{2}$ are Lagrange multipliers associated with the constraints on $\boldsymbol{\xi}_{1}$ and $\boldsymbol{\xi}_{2}$. The vectors $\boldsymbol{\xi}_{j}$ are eigenvectors of a linear Hermitian operator, and the Lagrange multipliers $\mu_{j}$ give a way of extending the dispersion relation to the nonlinear case in a coordinate-free way.

Formally solving this finite-dimensional Lagrangian system leads to the reduced Lagrangian

$$
\overline{\mathscr{L}}=\mu_{1}\left|A_{1}\right|^{2}+\mu_{2}\left|A_{2}\right|^{2}+\frac{1}{2} \sigma_{11}\left|A_{1}\right|^{4}+\sigma_{12}\left|A_{1}\right|^{2}\left|A_{2}\right|^{2}+\frac{1}{2} \sigma_{22}\left|A_{2}\right|^{4}+\cdots
$$

and to amplitude equations for $A_{1}$ and $A_{2}$ of the general form

$$
\begin{aligned}
& A_{1}\left(\mu\left(\omega_{1}, k_{1}\right)+\sigma_{11}\left|A_{1}\right|^{2}+\sigma_{12}\left|A_{2}\right|^{2}+\cdots\right)=0, \\
& A_{2}\left(\mu\left(\omega_{2}, k_{2}\right)+\sigma_{12}\left|A_{1}\right|^{2}+\sigma_{22}\left|A_{2}\right|^{2}+\cdots\right)=0 .
\end{aligned}
$$

To leading order, the Lagrange multipliers $\mu_{1}$ and $\mu_{2}$ are the dispersion relation for the linearized problem evaluated at $\left(\omega_{1}, k_{1}\right)$ and $\left(\omega_{2}, k_{2}\right)$, respectively. To compute the elements needed for the stability analysis, we need the functionals $\mathscr{A}_{j}$ and $\mathscr{B}_{j}$. To leading order they are

$$
\mathscr{A}_{j}(\omega, k)=-\frac{\partial}{\partial \omega_{j}} \mu_{j}(\omega, k)\left|A_{j}\right|^{2}+\cdots \quad \text { and } \quad \mathscr{B}_{j}(\omega, k)=-\frac{\partial}{\partial k_{j}} \mu_{j}(\omega, k)\left|A_{j}\right|^{2}+\cdots,
$$

where $(\omega, k):=\left(\omega_{1}, \omega_{2}, k_{1}, k_{2}\right)$. These expressions are verified by substituting (6.1) into the functionals (4.6). Using these expressions we compute

$$
\frac{\delta \mathscr{A}}{\delta \omega}=\left[\begin{array}{ll}
\frac{\partial \mathscr{A}_{1}}{\delta \omega_{1}} & \frac{\partial \mathscr{A}_{1}}{\delta \omega_{2}} \\
\frac{\partial \mathscr{A}_{2}}{\delta \omega_{1}} & \frac{\partial \mathscr{A}_{2}}{\delta \omega_{2}}
\end{array}\right]=\left[\begin{array}{cc}
\frac{\partial}{\partial \omega_{1}} a_{1}\left|A_{1}\right|^{2}+a_{1} \frac{\partial}{\partial \omega_{1}}\left|A_{1}\right|^{2}, & a_{1} \frac{\partial}{\partial \omega_{2}}\left|A_{1}\right|^{2} \\
a_{2} \frac{\partial}{\partial \omega_{1}}\left|A_{2}\right|^{2}, & \frac{\partial}{\partial \omega_{2}} a_{2}\left|A_{2}\right|^{2}+a_{2} \frac{\partial}{\partial \omega_{2}}\left|A_{2}\right|^{2}
\end{array}\right]+\cdots,
$$

where $a_{j}=-\frac{\partial}{\partial \omega_{j}} \mu_{j}, j=1,2$. Now apply the standing wave limit to this matrix,

$$
\omega_{2} \rightarrow \omega_{1}:=\omega, \quad k_{2} \rightarrow-k_{1}:=-k, \quad\left|A_{2}\right| \rightarrow\left|A_{1}\right|:=|A|,
$$

to find

$$
\frac{\delta \mathscr{A}}{\delta \omega}=-D_{\omega}^{2} \Lambda^{-1}+D_{\omega \omega}|A|^{2}\left(\begin{array}{ll}
1 & 0 \\
0 & 1
\end{array}\right)+\cdots
$$


where $D(\omega, k)=\lim _{\rightarrow \text { sWs }} \mu_{1}\left(\omega_{1}, k_{1}\right)=\lim _{\rightarrow \text { SWs }} \mu_{2}\left(\omega_{2}, k_{2}\right)$, and

$$
\Lambda:=\left[\begin{array}{ll}
a & b \\
b & a
\end{array}\right]=\lim _{\rightarrow \mathrm{SWs}}\left[\begin{array}{ll}
\sigma_{11} & \sigma_{12} \\
\sigma_{12} & \sigma_{22}
\end{array}\right] .
$$

Similarly we find

$$
\begin{gathered}
\frac{\delta \mathscr{A}}{\delta k}=-D_{\omega} D_{k} \Lambda^{-1}\left(\begin{array}{cc}
1 & 0 \\
0 & -1
\end{array}\right)+D_{\omega k}|A|^{2}\left(\begin{array}{cc}
1 & 0 \\
0 & -1
\end{array}\right)+\cdots \\
\frac{\delta \mathscr{B}}{\delta \omega}=\left(\frac{\delta \mathscr{A}}{\delta k}\right)^{T}=-D_{\omega} D_{k}\left(\begin{array}{cc}
1 & 0 \\
0 & -1
\end{array}\right) \Lambda^{-1}+D_{\omega k}|A|^{2}\left(\begin{array}{cc}
1 & 0 \\
0 & -1
\end{array}\right)+\cdots
\end{gathered}
$$

and so

$$
\frac{\delta \mathscr{A}}{\delta k}+\frac{\delta \mathscr{B}}{\delta \omega}=-\frac{2 a}{|\Lambda|} D_{\omega} D_{k}\left(\begin{array}{cc}
1 & 0 \\
0 & -1
\end{array}\right)+2 D_{\omega k}|A|^{2}\left(\begin{array}{cc}
1 & 0 \\
0 & -1
\end{array}\right)+\cdots
$$

For the third term in the matrix (5.7),

$$
\frac{\delta \mathscr{B}}{\delta k}=-D_{k}^{2} \frac{\Lambda}{|\Lambda|}+D_{k k}|A|^{2}\left(\begin{array}{cc}
1 & 0 \\
0 & 1
\end{array}\right)+\cdots
$$

Now, the stability quartic (5.8) in the standing wave limit takes the form

$$
\Delta(\lambda, \alpha)=a_{4} \lambda^{4}+a_{3} \lambda^{3}+a_{2} \lambda^{2}+a_{1} \lambda+a_{0}
$$

with

$$
\begin{aligned}
a_{4} & =\operatorname{det}\left(\frac{\delta A}{\delta \omega}\right)=\frac{D_{\omega}^{4}}{|\Lambda|}-\frac{2 a}{|\Lambda|} D_{\omega}^{2} D_{\omega \omega}|A|^{2}+\cdots \\
a_{3} & =\mathrm{i} \alpha \operatorname{Tr}\left(\frac{\delta A^{\#}}{\delta \omega}\left(\frac{\delta A}{\delta k}+\frac{\delta B}{\delta \omega}\right)\right)=0 \\
a_{2} & =-\alpha^{2} \operatorname{det}\left(\frac{\delta A}{\delta k}+\frac{\delta B}{\delta \omega}\right)-\alpha^{2} \operatorname{Tr}\left(\frac{\delta A^{\#}}{\delta \omega} \frac{\delta B}{\delta k}\right) \\
& =-\alpha^{2}\left(-\frac{2}{|\Lambda|} D_{\omega}^{2} D_{k}^{2}+\frac{2 a}{|\Lambda|}\left(-D_{\omega}^{2} D_{k k}-D_{k}^{2} D_{\omega \omega}+4 D_{\omega} D_{k} D_{\omega k}\right)|A|^{2}+\cdots\right) \\
a_{1} & =(\mathrm{i} \alpha)^{3} \operatorname{Tr}\left(\frac{\delta B}{\delta k}\left(\frac{\delta A}{\delta k}+\frac{\delta B}{\delta \omega}\right)\right)=0 \\
a_{0} & =\alpha^{4} \operatorname{det}\left(\frac{\delta B}{\delta k}\right)=\alpha^{4}\left(\frac{D_{k}^{4}}{|\Lambda|}-\frac{2 a}{|\Lambda|} D_{k}^{2} D_{k k}|A|^{2}+\cdots\right)
\end{aligned}
$$


Let

$$
\delta=-D_{\omega}^{2} D_{k k}-D_{k}^{2} D_{\omega \omega}+2 D_{\omega} D_{k} D_{\omega k}=\operatorname{det}\left[\begin{array}{ccc}
D_{\omega \omega} & D_{\omega k} & D_{\omega} \\
D_{k \omega} & D_{k k} & D_{k} \\
D_{\omega} & D_{k} & 0
\end{array}\right] .
$$

Then, to summarize, the stability quartic in the standing wave limit is

$\Delta(\lambda, \alpha)=\operatorname{det}\left[\lambda^{2} \frac{\delta \mathscr{A}}{\delta \omega}+\mathrm{i} \alpha \lambda\left(\frac{\delta \mathscr{A}}{\delta k}+\frac{\delta \mathscr{B}}{\delta \omega}\right)+(\mathrm{i} \alpha)^{2} \frac{\delta \mathscr{B}}{\delta k}\right]=a_{4} \lambda^{4}+a_{2}(\mathrm{i} \alpha)^{2} \lambda^{2}+a_{0}(\mathrm{i} \alpha)^{4}$

with

$$
\begin{aligned}
& a_{4}=+\frac{D_{\omega}^{4}}{|\Lambda|}-\frac{2 a}{|\Lambda|} D_{\omega}^{2} D_{\omega \omega}|A|^{2}+\cdots \\
& a_{2}=-\frac{2}{|\Lambda|} D_{\omega}^{2} D_{k}^{2}+\frac{2 a}{|\Lambda|}\left(\delta+2 D_{\omega} D_{k} D_{\omega k}\right)|A|^{2}+\cdots \\
& a_{0}=+\frac{D_{k}^{4}}{|\Lambda|}-\frac{2 a}{|\Lambda|} D_{k}^{2} D_{k k}|A|^{2}+\cdots
\end{aligned}
$$

Apply the stability-instability classification in section 5.1, which requires the expressions

$$
\begin{aligned}
a_{0} a_{4} & =\frac{D_{\omega}^{4} D_{k}^{4}}{|\Lambda|^{2}}+\cdots>0, \\
-a_{2} a_{4} & =\frac{2}{|\Lambda|^{2}} D_{k}^{2} D_{\omega}^{6}+\cdots>0, \\
a_{2}^{2}-4 a_{0} a_{4} & =-16 a \delta \frac{D_{\omega}^{2} D_{k}^{2}}{|\Lambda|^{2}}|A|^{2}+\cdots .
\end{aligned}
$$

Hence, from the stability-instability classification in section 5.1, if we assume the conditions

$$
D_{\omega} \neq 0, \quad D_{k} \neq 0, \quad \operatorname{det}(\Lambda) \neq 0, \quad a \neq 0, \quad \text { and } \quad \delta \neq 0
$$

are satisfied, we can conclude, for $|A|$ sufficiently small, that the stability quartic (5.7) has an unstable eigenvalue if and only if

$$
a \delta>0 .
$$

A significant feature of this result is that the instability of standing waves is independent of the standing wave frequency correction. To see this, go back to (6.3) and take the standing wave limit,

$$
\begin{aligned}
& A_{1}\left(D(\omega, k)+a\left|A_{1}\right|^{2}+b\left|A_{2}\right|^{2}+\cdots\right)=0, \\
& A_{2}\left(D(\omega, k)+b\left|A_{1}\right|^{2}+a\left|A_{2}\right|^{2}+\cdots\right)=0 .
\end{aligned}
$$

Hence

$$
\begin{aligned}
& \omega^{T W}=\omega_{0}-\frac{a}{D_{\omega}}\left|A_{1}\right|^{2}+\cdots, \quad A_{2}=0, \\
& \omega^{S W}=\omega_{0}-\frac{(a+b)}{D_{\omega}}\left|A_{1}\right|^{2}+\cdots, \quad A_{2}=A_{1} .
\end{aligned}
$$




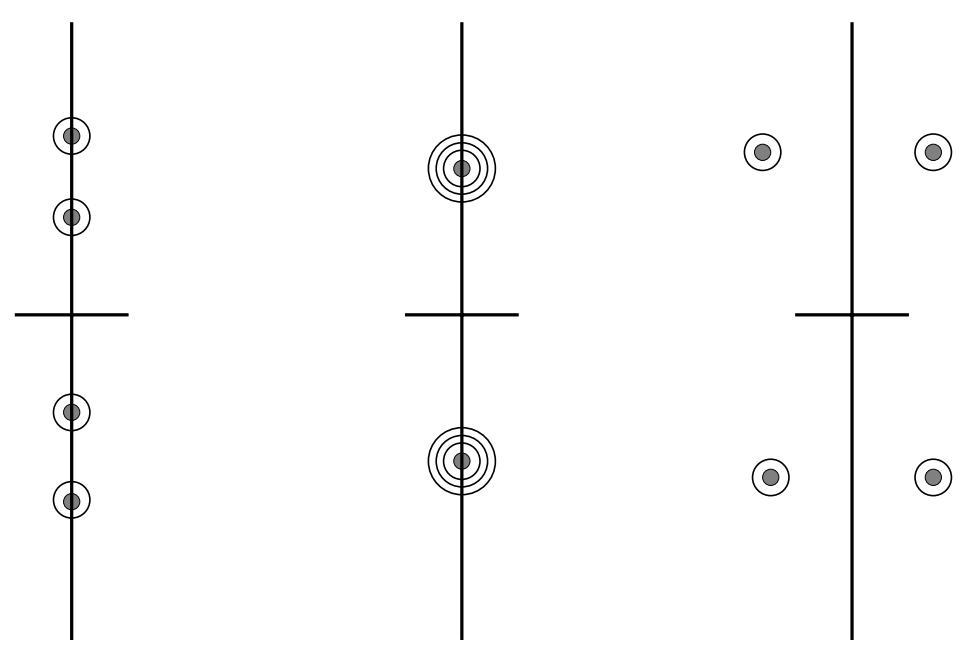

FIG. 2. Schematic of the position of the eigenvalues at the threshold of instability for weakly nonlinear standing waves.

Although the weakly nonlinear correction to the frequency for standing waves depends on $b$, the modulation instability at low amplitude is independent of $b$. Effectively, the stability problem decouples at low amplitude.

To confirm that a weakly nonlinear traveling wave has the same instability condition as the standing wave, we can compare $a \delta>0$ with the Whitham condition. If $D\left(\omega_{0}(k), k\right)=0$ and $D_{\omega} \neq 0$, then $D_{\omega} \omega_{0}^{\prime}(k)+D_{k}=0$ and so

$$
\omega_{0}^{\prime \prime}(k)=\frac{1}{D_{\omega}^{3}} \operatorname{det}\left[\begin{array}{ccc}
D_{\omega \omega} & D_{\omega k} & D_{\omega} \\
D_{k \omega} & D_{k k} & D_{k} \\
D_{\omega} & D_{k} & 0
\end{array}\right]=\frac{1}{D_{\omega}^{3}} \delta .
$$

From the above expression for the frequency of the traveling waves, $\omega^{T W}$, we have that $\omega_{2}=-a / D_{\omega}$ and so

$$
\operatorname{sign}(a \delta)=\operatorname{sign}\left(-\omega_{2} D_{\omega} \omega_{0}^{\prime \prime}(k) D_{\omega}^{4}\right)=-\operatorname{sign}\left(\omega_{0}^{\prime \prime}(k) \omega_{2}\right),
$$

showing that $a \delta>0$ is equivalent to $\omega_{0}^{\prime \prime}(k) \omega_{2}<0$.

This instability condition for weakly nonlinear standing waves agrees with the instability condition derived by Knobloch and Pierce [18], obtained from the coupled NLS equations with mean-field terms for counterpropagating waves.

For finite-amplitude standing waves, the instability of standing waves will in general differ from the instability of traveling waves. It is an interesting open problem to determine precisely how this instability will change for finite amplitude standing waves. It can be studied either by carrying the amplitude expansion to the next order or numerically.

There is another subtle difference between the traveling wave instability and standing wave instability, which shows up even for weakly nonlinear standing waves. For the standing wave, the unstable subspace is twice as large as the case of traveling waves. A schematic is shown in Figure 2. This figure shows the temporal eigenvalues of the linear stability problem for weakly nonlinear standing waves, with 
instability arising through a collision of eigenvalues of opposite signature. Note that each eigenvalue is double, and so the unstable subspace is four-dimensional (whereas for traveling waves it is two-dimensional). The multiple eigenvalues will not persist for finite-amplitude standing waves, suggesting that the behavior of the instability for finite-amplitude standing waves will be more dramatic than traveling waves in general and weakly nonlinear standing waves in particular.

6.1. Example: Calculations for a scalar nonlinear wave equation. An elementary example of the theory is obtained by considering the scalar nonlinear wave equation: (1.1) with $m=1$. Let $V(u)$ be any smooth function with leading Taylor expansion

$$
V(u)=\frac{1}{2} a_{1} u^{2}+\frac{1}{3} a_{2} u^{3}+\frac{1}{4} a_{3} u^{4}+\cdots, \quad a_{1}>0 .
$$

Then a straightforward calculation leads to the reduced Lagrangian

$$
\overline{\mathscr{L}}=\mu_{1}\left|A_{1}\right|^{2}+\mu_{2}\left|A_{2}\right|^{2}+\frac{1}{2} \sigma_{11}|A|^{4}+\sigma_{12}\left|A_{1}\right|^{2}\left|A_{2}\right|^{2}+\frac{1}{2} \sigma_{22}\left|A_{2}\right|^{4}+\cdots
$$

with $\mu_{j}(\omega, k)=k_{j}^{2}-\omega_{1}^{2}+a_{1}, \sigma_{11}=\sigma_{22}=-\frac{5}{3 a_{1}} a_{2}^{2}+\frac{9}{4} a_{3}$, and

$\sigma_{12}=-2 a_{2}^{2}\left(\frac{1}{a_{1}}+\frac{1}{a_{1}-\left(\omega_{1}+\omega_{2}\right)^{2}+\left(k_{1}+k_{2}\right)^{2}}+\frac{1}{a_{1}-\left(\omega_{1}-\omega_{2}\right)^{2}+\left(k_{1}-k_{2}\right)^{2}}\right)+3 a_{3}$.

Computing the parameter Jacobian (4.8) and taking the limit $\omega_{2} \rightarrow \omega_{1}:=\omega$ and $k_{2} \rightarrow-k_{1}:=-k$ we find

$$
D(\omega, k)=k^{2}-\omega^{2}+a_{1}, \quad \delta=-8 a_{1}, \quad \text { and } \quad a=\lim _{\rightarrow \mathrm{SWs}} \sigma_{11}=\sigma_{11},
$$

and so $a \delta=-8 a_{1}\left(-\frac{5}{3 a_{1}} a_{2}^{2}+\frac{9}{4} a_{3}\right)$. Hence both traveling waves and standing wave solutions of (1.1) are unstable in the weakly nonlinear limit whenever

$$
20 a_{2}^{2}-27 a_{1} a_{3}>0 \text { and } a_{1}>0 .
$$

The instability of finite-amplitude standing wave solutions of even this scalar nonlinear wave equation is an open problem, but the theory of this paper can be applied, given (either numerical or analytic) expressions for the finite-amplitude standing waves.

7. Small divisors and the equivariant Lyapunov center theorem. The obstacle to a rigorous proof of the existence of smooth families of standing waves and the linear stability theory is a potential small divisor problem. This issue can be illustrated by considering the scalar version of the nonlinear wave equation (1.1), which can be written

$$
u_{t t}-u_{x x}+a_{1} u=V^{\prime}(u)-a_{1} u,
$$

where $a_{1}=V^{\prime \prime}(0)$ is some positive real number. In application of the implicit function theorem to the existence of standing waves, linear systems of the type of the left-hand side have to be inverted on the complement of its kernel, on a space of space-time periodic functions. Such systems can be written in the general form

$$
V_{t}=\mathbf{L} V+\mathbf{f}(x, t), \quad \mathbf{L}=\left[\begin{array}{cc}
0 & I \\
\partial_{x x}-a_{1} & 0
\end{array}\right],
$$


where $\mathbf{f}(x, t)$ is a vector-valued periodic function of $x$ and $t$. Now, the spectrum of $\mathbf{L}$ on a space of $2 \pi$-periodic functions is

$$
\lambda_{n}=\mathrm{i} \sqrt{n^{2}+a_{1}}:=\mathrm{i} \omega_{n}, \quad n \in \mathbb{Z} .
$$

The spectrum consists of a countable number of purely imaginary eigenvalues. Now, $a_{1}$ can be chosen so that

$$
\lambda_{1} j-\lambda_{n} \neq 0, \quad j \geq 1, n \neq 1,
$$

which is the usual nonresonance condition of the Lyapunov center theorem. However, the distance $\left|\omega_{1} j-\omega_{n}\right|$ may tend to zero as $j, n$ tend to infinity, creating a small divisor problem. In other words, a frequency $\omega_{n}$ when $n$ is large enough may get arbitrarily close to a resonant multiple of $\omega_{1}$.

Effectively, what is needed is a version of the Lyapunov center theorem in infinite dimensions. The first result of this type is due to Craig and Wayne [12] and uses NashMoser theory to overcome the small divisor problem. However, the resulting branches of periodic solutions are not smooth but lie on a Cantor-like subset of parameter space.

By imposing the stronger diophantine condition on the frequencies

$$
\left|\omega j-\omega_{n}\right| \geq \frac{\gamma}{j}, \quad j \geq 1, \quad n \geq 2, \quad \text { for some } \gamma>0,
$$

Bambusi [1] and Bambusi and Paleari [4] prove that the ordinary implicit function can be used, and this leads to partial smoothness of branches of periodic solutions.

These results are encouraging, but they still do not provide sufficient smoothness for the limits required in section 5. Moreover, the present analysis uses symmetry in a central way, and so an equivariant version of the Lyapunov center theorem [22] generalized to infinite dimensions would be required. Some intriguing results in this direction are given by Bambusi and Gaeta [3].

8. Instability of standing water waves. One of the most interesting examples of standing waves is standing water waves. These waves are most commonly observed and studied in the context of sloshing of fluid in a vessel. However, they are also a central part of pattern formation in the open ocean. The first nonlinear theory for standing waves was proposed by Rayleigh [27]. Indeed, he showed that they arise naturally along with traveling waves in any analysis of weakly nonlinear space and time periodic water waves. Since Rayleigh's work there has been a wide range of analytical and numerical theories for standing waves; see [11] for a list of references.

Recently, progress has been made in developing a rigorous theory for existence of standing waves. In finite depth small divisors arise, and a rigorous proof in this case for weakly nonlinear standing waves has been given by Plotnikov and Toland [26]. The proof uses a Nash-Moser framework, and therefore the branches of periodic solutions are not smooth, certainly not smooth enough to embed them in a higher parameter family. Surprisingly, the problem in infinite depth is more difficult. The kernel of the linearized problem is infinite-dimensional and the dispersion relation is algebraic, but recent significant progress has been made $[14,15]$.

For weakly nonlinear standing waves, stability results have been reported by Okamura [23] and Knobloch and Pierce [18] using modulation equations. The paper [18] gives the first correct analytic result for instability of weakly nonlinear standing waves. For finite-amplitude standing waves, the only results in the literature on the linear 
stability is the work of Mercer and Roberts [21]. There are several interesting results in [21]. They compute long-wave instabilities at finite amplitude (called subharmonic instabilities there). They compute the action $\left(\mathscr{A}_{1}\right.$ here) as a function of frequency $\left(\omega_{1}\right.$ here) and show that there is a point where $\partial_{\omega_{1}} \mathscr{A}_{1}=0$. This latter point will have an effect on the modulation instability at that point.

The theory of this paper suggests a new approach to the numerical computation of standing waves. The standing waves should be embedded in a four-parameter family and then the elements of (4.8) computed to study a wider range of stability properties. This embedding would not increase computation time (for standing waves or four-parameter two-wave interaction, the solution is expanded in a double Fourier series) but would increase the range of parameter space. However, it is only the parameter space near the standing waves that is of interest, and the computation of the functionals and their parameter dependence is a secondary calculation.

On a formal level one can draw a number of conclusions about the instability of standing water waves from the theory reported in this paper. First, the water-wave problem can be formulated as a multisymplectic system $[6,7]$ and the framework of this paper applied. For weakly nonlinear standing waves the conclusion for deep water is immediate: weakly nonlinear standing waves are unstable to a Benjamin-Feir instability in the same way that traveling waves are unstable. This is in agreement with the results of [18]. Further numerical calculations would be needed to test the theory of this paper at large amplitude to compare with and extend the results of [21].

The case of standing waves in finite depth may also have mean flow generation. For traveling waves, it is well known that reducing the depth creates a mean flow that stabilizes the Benjamin-Feir instability. Therefore an interesting open problem would be to determine the effect of mean flow on the stability of standing water waves in finite depth. Results obtained using modulation equations by Knobloch and Pierce [18] suggest that the weakly nonlinear finite-depth standing waves are affected by mean flow in exactly the same way as traveling waves. However, the role of mean flow in the stability of finite-amplitude standing waves is an open question.

9. Concluding remarks. The basic strategy here - embed a multiperiodic, say, $N$-periodic, pattern in an $N$-wave interaction with $2 N$ parameters, compute parameter Jacobians, then take a limit to the original $N$-parameter wave to obtain stability information - has wider applicability. For example, in [10] this idea is generalized to determine stability conditions for short-crested Stokes waves in three space dimensions.

Short-crested Stokes waves are solutions of the form

$$
Z(x, y, t)=\widehat{Z}\left(\theta_{1}, \theta_{2}\right), \quad \theta_{1}=k x+\ell y+\omega t, \quad \theta_{2}=k x-\ell y+\omega t .
$$

They are three-parameter doubly periodic solutions and have been widely studied by oceanographers and engineers because they arise as a secondary bifurcation from classical Stokes waves and are known to influence sand transport. Short-crested waves are a generalization of standing waves in the sense that they can be characterized as synchronized oblique traveling waves, and in the limit as the angle between the two waves becomes zero they reduce to standing waves.

There are a number of open questions in the fluid mechanics literature about their stability. The theory of this paper generalizes to this problem in a straightforward way. The short-crested wave is embedded in the six-parameter two-phase wavetrain $Z(x, y, t)=\widehat{Z}\left(\theta_{1}, \theta_{2}\right)$ but with

$$
\theta_{j}=\omega_{j} t+k_{j} x+\ell_{j} y+\theta_{j}^{o}, \quad j=1,2 .
$$


These waves, when characterized by a constrained variational principle, generate the $6 \times 6$ matrix

$$
\left(\begin{array}{lll}
\frac{\delta \mathscr{A}}{\delta \omega} & \frac{\delta \mathscr{A}}{\delta k} & \frac{\delta \mathscr{A}}{\delta \ell} \\
\frac{\delta \mathscr{B}}{\delta \omega} & \frac{\delta \mathscr{B}}{\delta k} & \frac{\delta \mathscr{B}}{\delta \ell} \\
\frac{\delta \mathscr{C}}{\delta \omega} & \frac{\delta \mathscr{C}}{\delta k} & \frac{\delta \mathscr{C}}{\delta \ell}
\end{array}\right) .
$$

Proceeding as in section 5, a stability theory can be developed that predicts all longwave instabilities using the entries of the above matrix. The complete details are given elsewhere [10].

Another generalization of interest is the study of the stability of hexagonal ocean patterns by embedding them in a three-phase wave train - with nine parameters - and then following the strategy in this paper to develop a theory for long-wave instability.

Appendix A. $O(2)$-invariant Hamiltonian systems and the spherical pendulum. Some insight into the geometry of nonlinear wave equations on the real line with periodic boundary conditions and $x$-reflection symmetry can be gained by examining the finite-dimensional analogue. This analogy is useful for illustrating the toral structure, but the analogy can be taken only so far, since the most interesting consequence for nonlinear wave equations - the geometry of modulational instability is absent in finite dimensions.

Consider a Hamiltonian system on $\mathbb{R}^{4}$ with standard symplectic operator $\mathbf{J}$,

$$
\mathbf{J} U_{t}=\nabla H(U), \quad U \in \mathbb{R}^{4},
$$

and suppose $H$ is smooth and the system is $\mathbf{O}(2)$-equivariant. That is, there is a representation $\Gamma$ of $\mathbf{O}(2)$ acting on $\mathbb{R}^{4}$ such that $H$ is $\Gamma$-invariant and the action of $\Gamma$ is symplectic [22]. Near a $\Gamma$-invariant equilibrium there are generically two classes of periodic solutions: traveling waves and standing waves [22].

The canonical example of an $\mathbf{O}(2)$-equivariant Hamiltonian system is the spherical pendulum (see $[22,5]$ ), and it is sufficient to restrict attention to this example. For the spherical pendulum, the geometry and nature of solutions can be seen explicitly. The traveling waves are the conical pendulum solutions, and standing waves are the planar pendulum solutions. There are two traveling waves (one rotating clockwise and one rotating counterclockwise), and there is an $\mathbf{S O}(2)$ orbit of standing waves (the plane of motion of the planar pendulum can be rotated around). The standing waves of the spherical pendulum have zero angular momentum.

There is another well-known class of solutions of the spherical pendulum: the toral solutions which have a smoothly varying rotation number and nonzero angular momentum. These are sometimes called relative periodic orbits. Physically, they correspond to a precessing planar pendulum.

The solutions of the spherical pendulum can be usefully viewed in the energymomentum space, as shown in Figure 3, where $E$ represents the value of the energy and $I$ represents the value of the momentum. The standing waves are along the $I=0$ axis. The traveling waves correspond to minima of the energy restricted to level sets of the momentum and lie along the two curves shown. There are no solutions associated with $(I, E)$ values below the traveling wave curves, and the region between the two curves excluding $I=0$ is filled with toral solutions with smoothly varying rotation number. 


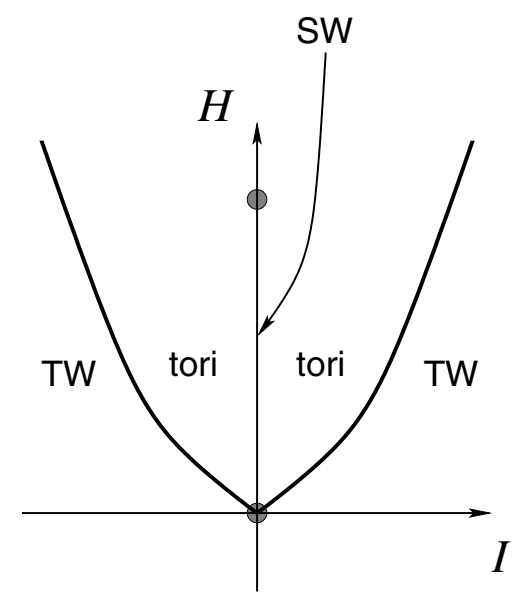

FIG. 3. Schematic of the energy-momentum space for the spherical pendulum. The two highlighted points on the line of zero momentum are the two equilibria (vertical up and vertical down) of the spherical pendulum.

A periodic solution, of period $T=\frac{2 \pi}{\omega}$, of a finite-dimensional Hamiltonian system such as (A.1) can be characterized by a variational principle: a critical point of $H$ restricted to level sets of the action,

$$
\mathscr{A}=\frac{1}{2 \pi} \int_{0}^{2 \pi} A(U) \mathrm{d} \theta, \quad A(U)=\frac{1}{2}\left\langle\mathbf{J} U_{\theta}, U\right\rangle
$$

with the frequency, $\omega$, a Lagrange multiplier. The Lagrange necessary condition for this variational principle is

$$
\nabla H-\omega \nabla A=\nabla H-\omega \mathbf{J} U_{\theta}=0, \quad \theta=\omega t+\theta^{\circ} .
$$

A standing wave state would have the additional requirement that it is invariant under reflection (the reflection subgroup of $\mathbf{O}(2)$ ).

Now we come to the main point of this section. Can the limit $I \rightarrow 0$ be taken in the class of toral solutions leading to a standing wave?

Consider embedding the standing wave in a toral solution. Let $U(t)=\widehat{U}\left(\theta_{1}, \theta_{2}\right)$ with $\theta_{j}=\omega_{j} t+\theta_{j}^{o}$ for $j=1,2$. Then a variational characterization is again natural, and the Lagrange necessary condition is

$$
\nabla H-\omega_{1} \nabla A_{1}-\omega_{2} \nabla A_{2}=\nabla H(\widehat{Z})-\omega_{1} \mathbf{J} \partial_{\theta_{1}} \widehat{U}-\omega_{2} \mathbf{J} \partial_{\theta_{2}} \widehat{U}=0, \quad \widehat{U}: \mathbb{T}^{2} \rightarrow \mathbb{R}^{4} .
$$

It follows from standard Lagrange multiplier theory that a state satisfying (A.3) is nondegenerate when

$$
\operatorname{det}\left[\begin{array}{ll}
\frac{\partial \omega_{1}}{\partial I_{1}} & \frac{\partial \omega_{1}}{\partial I_{2}} \\
\frac{\partial \omega_{2}}{\partial I_{1}} & \frac{\partial \omega_{2}}{\partial I_{2}}
\end{array}\right] \neq 0,
$$

where $I_{1}$ and $I_{2}$ represent values of the two actions. Now, solutions of this variational principle are smooth functions of the frequencies, away from the singularities (the 
two equilibrium points and the two branches of traveling waves). This can be seen explicitly using the integrability of the spherical pendulum [13], but it follows more generally from symmetry. The more surprising smoothness result is that the frequency map (A.4) exists - in the limit from a toral state to the line $I=0$ - and is well defined (away from the two equilibrium points). Suppose $I_{2}$ represents the angular momentum in (A.4); then Horosov [13] proves that

$$
\lim _{I_{2} \rightarrow 0} \operatorname{det}\left[\begin{array}{ll}
\frac{\partial \omega_{1}}{\partial I_{1}} & \frac{\partial \omega_{1}}{\partial I_{2}} \\
\frac{\partial \omega_{2}}{\partial I_{1}} & \frac{\partial \omega_{2}}{\partial I_{2}}
\end{array}\right]=\operatorname{det}\left[\begin{array}{cc}
\left(\frac{\partial \omega_{1}}{\partial I_{1}}\right)^{0} & 0 \\
0 & \left(\frac{\partial \omega_{2}}{\partial I_{2}}\right)^{0}
\end{array}\right]=\left(\frac{\partial \omega_{1}}{\partial I_{1}}\right)^{0}\left(\frac{\partial \omega_{2}}{\partial I_{2}}\right)^{0} \neq 0 .
$$

Although the limit $I_{2} \rightarrow 0$ results in a degeneration from a toral solution to a periodic solution, the toral frequency map does not degenerate! This nondegeneracy arises because the planar pendulum solutions lie on a torus and so the tangent space of the manifold of standing waves is two-dimensional. The first term in the product on the right-hand side of (A.5) is the change in frequency with amplitude (or energy) of the planar pendulum, and the second term in the product just says that the second frequency changes smoothly in going from negative to positive angular momentum (or vice versa).

The above geometry is also central to the standing wave problem associated with nonlinear wave equations on the real line. In the second variational principle in section 4, the standing wave is being embedded in a generalized multisymplectic relativeperiodic orbit. In other words, geometrically there should be a smooth variation of the parameters. This would be true of any finite-dimensional approximation of the standing wave problem. In the limit as the number of modes goes to infinity, the small divisor issue again appears.

Appendix B. Multisymplectic Noether theory and momentum conservation. In this appendix, classical Noether theory is applied to the Lagrangian in the canonical multisymplectic form (1.6). A Lagrangian $\mathscr{L}=\iint \mathrm{L}\left(Z, Z_{t}, Z_{x}\right) \mathrm{d} x \wedge \mathrm{d} t$, with $Z(x, t)$ vector valued, which does not depend explicitly on $x$, has a symmetry with generator $\mathbf{v}=\frac{\partial}{\partial x}$. Using Noether's theorem [24, section 4.4], this symmetry generates a conservation law,

$$
\mathrm{I}(Z)_{t}+\mathrm{P}(Z)_{x}=0
$$

with

$$
\mathrm{I}(Z)=\left\langle Z_{x}, \frac{\partial \mathrm{L}}{\partial Z_{t}}\right\rangle \quad \text { and } \quad \mathrm{P}(Z)=\left\langle Z_{x}, \frac{\partial \mathrm{L}}{\partial Z_{x}}\right\rangle-\mathrm{L}\left(Z, Z_{t}, Z_{x}\right)
$$

where $\langle\cdot, \cdot\rangle$ is the standard inner product on $\mathbb{R}^{n}$. Applying these formulas to $\mathrm{L}$ in canonical form,

$$
\mathrm{L}\left(Z, Z_{t}, Z_{x}\right)=\frac{1}{2}\left\langle\mathbf{M} Z_{t}, Z\right\rangle+\frac{1}{2}\left\langle\mathbf{K} Z_{x}, Z\right\rangle-S(Z),
$$

leads to

$$
\mathrm{I}(Z)=\frac{1}{2}\left\langle\mathbf{M} Z_{x}, Z\right\rangle, \quad \mathrm{P}(Z)=S(Z)-\frac{1}{2}\left\langle\mathbf{M} Z_{t}, Z\right\rangle
$$


This conservation law can be confirmed by direct calculation,

$$
\begin{aligned}
\mathrm{I}(Z)_{t} & =\frac{1}{2}\left\langle\mathbf{M} Z_{x t}, Z\right\rangle+\frac{1}{2}\left\langle\mathbf{M} Z_{x}, Z_{t}\right\rangle \\
& \left.=\frac{\partial}{\partial x}\left(\frac{1}{2}\left\langle\mathbf{M} Z_{t}, Z\right\rangle\right)-\left\langle\mathbf{M} Z_{t}, Z_{x}\right\rangle \quad \text { (using skew-symmetry of } \mathbf{M}\right) \\
& \left.=\frac{\partial}{\partial x}\left(\frac{1}{2}\left\langle\mathbf{M} Z_{t}, Z\right\rangle\right)-\left\langle\nabla S(Z)-\mathbf{K} Z_{x}, Z_{x}\right\rangle \quad \text { (substituting for } \mathbf{M} Z_{t} \text { using }(3.1)\right) \\
& =\frac{\partial}{\partial x}\left(\frac{1}{2}\left\langle\mathbf{M} Z_{t}, Z\right\rangle-S(Z)\right) \quad(\text { using skew-symmetry of } \mathbf{K})
\end{aligned}
$$

and hence $\mathrm{I}(Z)_{t}+\left(S(Z)-\frac{1}{2}\left\langle\mathbf{M} Z_{t}, Z\right\rangle\right)_{x}=0$.

\section{REFERENCES}

[1] D. Bambusi, Lyapunov center theorem for some nonlinear PDEs: A simple proof, Ann. Scuola Norm. Sup. Pisa Cl. Sci. (4), 29 (2000), pp. 823-837.

[2] D. Bambusi, A. Carati, And A. Ponno, The nonlinear Schrödinger equation as a resonant normal form, Discrete Contin. Dyn. Syst. Ser. B, 2 (2002), pp. 109-128.

[3] D. Bambusi And C. Gaeta, On the persistence of invariant tori and a theorem of Nekhoroshev, Math. Phys. Electron. J., 8 (2002), Paper 1.

[4] D. Bambusi And S. Paleari, Families of periodic solutions of resonant PDEs, J. Nonlinear Sci., 11 (2001), pp. 69-87.

[5] L. M. Bates And R. H. Cushman, Global Aspects of Classical Integrable Systems, BirkhäuserVerlag, Basel, 1997.

[6] T. J. BRIDGes, Periodic patterns, linear instability, symplectic structure and mean-flow dynamics for 3D surface waves, Philos. Trans. Roy. Soc. London Ser. A, 354 (1996), pp. 533-574.

[7] T. J. BRIdges, Multi-symplectic structures and wave propagation, Math. Proc. Cambridge Philos. Soc., 121 (1997), pp. 147-190.

[8] T. J. BRidges, F. DiAs, AND D. Menasce, Steady three-dimensional water-wave patterns on a finite-depth fluid, J. Fluid Mech., 436 (2001), pp. 145-175.

[9] T. J. Bridges and F. E. Laine-Pearson, Multisymplectic relative equilibria, multiphase wavetrains, and coupled NLS equations, Stud. Appl. Math., 107 (2001), pp. 137-155.

[10] T. J. Bridges and F. E. Laine-Pearson, The Long-Wave Instability of Short-Crested Waves, via Embedding in the Oblique Two-Wave Interaction, submitted.

[11] P. J. Bryant and M. Stiassne, Different forms for nonlinear standing waves in deep water, J. Fluid Mech., 272 (1994), pp. 135-156.

[12] W. Craig And C. E. WAYne, Newton's method and periodic solutions of nonlinear wave equations, Comm. Pure Appl. Math., 46 (1993), pp. 1409-1501.

[13] E. Horosov, Perturbations of the spherical pendulum and Abelian integrals, J. Reine Angew. Math., 408 (1990), pp. 114-135.

[14] G. Iooss, Semi-analytic theory of standing waves in deep water for several dominant modes, R. Soc. Lond. Proc. Ser. A Math. Phys. Eng. Sci., 455 (1999), pp. 3513-3526.

[15] G. Iooss, On the standing wave problem in deep water, J. Math. Fluid Mech., 4 (2002), pp. 155-185.

[16] P. Kirrmann, G. Schneider, And A. Mielke, The validity of modulation equations for extended systems with cubic nonlinearities, Proc. Roy. Soc. Edinburgh Sect. A, 122 (1992), pp. 85-91.

[17] E. Knobloch and J. D. GibBon, Coupled NLS equations for counter propagating waves in systems with reflection symmetry, Phys. Lett. A, 145 (1991), pp. 353-356.

[18] E. Knobloch and R. D. Pierce, On the modulational instability of travelling and standing water waves, Phys. Fluids, 6 (1994), pp. 1177-1190.

[19] S. B. KuKsin, Analysis of Hamiltonian PDEs, Oxford University Press, Oxford, UK, 2000.

[20] J. Mawhin And M. Willem, Critical Point Theory and Hamiltonian Systems, Springer-Verlag, Berlin, 1989.

[21] G. N. Mercer And A. J. Roberts, Standing waves in deep water. Their stability and extreme form, Phys. Fluids A, 4 (1992), pp. 259-269. 
[22] J. A. Montaldi, R. M. Roberts, and I. N. Stewart, Periodic solutions near equilibria of symmetric Hamiltonian systems, Philos. Trans. Roy. Soc. London Ser. A, 325 (1988), pp. 237-293.

[23] M. OKamura, Instabilities of weakly nonlinear standing gravity waves, J. Phys. Soc. Japan, 53 (1984), pp. 3788-3796.

[24] P. J. Olver, Application of Lie Groups to Differential Equations, Springer-Verlag, New York, 1986.

[25] R. D. Pierce And C. E. WAyne, On the validity of mean-field amplitude equations for counterpropagating wavetrains, Nonlinearity, 8 (1995), pp. 769-799.

[26] P. I. Plotnikov and J. F. Toland, Nash-Moser theory for standing water waves, Arch. Ration. Mech. Anal., 159 (2001), pp. 1-83.

[27] LoRd RAYleigh, Deep water waves, progressive or stationary, to the third order approximation, Proc. Roy. Soc. London A, 91 (1915), pp. 345-353.

[28] C. Sulem And P.-L. Sulem, The Nonlinear Schrödinger Equation. Self-Focusing and Wave Collapse, Appl. Math. Sci. 139, Springer-Verlag, New York, 1999.

[29] G. B. Whitham, Linear and Nonlinear Waves, Wiley-Interscience, New York, 1974.

[30] L. V. Yakushevich, Nonlinear Physics of DNA, 2nd rev. ed., Wiley-VCH, Weinheim, Germany, 2004 . 